\title{
Evidence of Physiotherapy Interventions for Patients with Chronic Neck Pain: A Systematic Review of Randomised Controlled Trials
}

\author{
Pia Damgaard, ${ }^{1,2}$ Else Marie Bartels, ${ }^{3}$ Inge Ris, ${ }^{1}$ \\ Robin Christensen, ${ }^{1,3}$ and Birgit Juul-Kristensen ${ }^{1,4}$ \\ ${ }^{1}$ Research Unit of Musculoskeletal Function and Physiotherapy, Institute of Sports Science and Clinical Biomechanics, \\ University of Southern Denmark, Campusvej 55, 5230 Odense M, Denmark \\ ${ }^{2}$ Department of Rehabilitation, Aeroe Municipality, 5970 Aeroeskoebing, Denmark \\ ${ }^{3}$ The Parker Institute, Department of Rheumatology, Copenhagen University Hospital, 2000 Frederiksberg, Copenhagen, Denmark \\ ${ }^{4}$ Bergen University College, Institute of Occupational Therapy, Physiotherapy and Radiography, Department of Health Sciences, \\ 5020 Bergen, Norway
}

Correspondence should be addressed to Birgit Juul-Kristensen; bjuul-kristensen@health.sdu.dk

Received 12 February 2013; Accepted 13 March 2013

Academic Editors: A. Blumenfeld and A. Nackley

Copyright (C) 2013 Pia Damgaard et al. This is an open access article distributed under the Creative Commons Attribution License, which permits unrestricted use, distribution, and reproduction in any medium, provided the original work is properly cited.

\begin{abstract}
Chronic neck pain (CNP) is common and costly, and the effect of physiotherapeutic interventions on the condition is unclear. We reviewed the literature for evidence of effect of physiotherapy interventions on patients with CNP. Five bibliographic databases (MEDLINE, EMBASE, CINAHL, Cochrane Library, and PEDro) were systematically searched. Randomised, placebo and activetreatment-controlled trials including physiotherapy interventions for adults with CNP were selected. Data were extracted primary outcome was pain. Risk of bias was appraised. Effect of an intervention was assessed, weighted to risk of bias. 42 trials reporting on randomised comparisons of various physiotherapy interventions and control conditions were eligible for inclusion involving 3919 patients with CNP. Out of these, 23 were unclear or at high risk of bias, and their results were considered moderate- or lowquality evidence. Nineteen were at low risk of bias, and here eight trials found effect on pain of a physiotherapy intervention. Only exercise therapy, focusing on strength and endurance training, and multimodal physiotherapy, cognitive-behavioural interventions, massage, manipulations, laser therapy, and to some extent also TNS appear to have an effect on CNP. However, sufficient evidence for application of a specific physiotherapy modality or aiming at a specific patient subgroup is not available.
\end{abstract}

\section{Introduction}

Musculoskeletal disorders are threatening quality of life by having the potential to restrict daily activities, cause absence from work, and result in a change or discontinuation in employment. These disorders are expensive for society and for patients and are responsible for the highest number of healthy years lost [1-4]. The prevalence of chronic neck pain varies. The 12-month prevalence of pain typically ranges between $30 \%$ and $50 \%$; the 12 -month prevalence of activitylimiting pain is $1.7 \%$ to $11.5 \%$ [5]. The annual incidence of neck pain associated with whiplash varies greatly. Although 50\% of whiplash victims recover in three to six months, $30 \%$ to $40 \%$ have persisting mild to moderate pain and $10 \%$ to $20 \%$ retain more severe pain [6]. It is a multifaceted phenomenon with physical impairment, psychological distress, and social dysfunction, which calls for an evidence-based, cost-effective rehabilitation treatment [7-11].

According to a Dutch study, $44 \%$ of patients with chronic neck pain visited their general practitioner (GP) with the condition during a twelve-month period; $51 \%$ of these were referred to physiotherapy treatment [12]. Knowledge of the actual effect of physiotherapy is therefore important and is anticipated to be reflected in the awareness of evidence-based practice among physiotherapists.

The Cochrane Collaboration has provided systematic reviews on the effect of massage for mechanical neck disorders [13], patient education for neck pain [14], electrotherapy 
for neck pain [15], mechanical traction for neck pain with or without radiculopathy [16], and conservative treatment for whiplash [17]. The overall conclusion has been that the evidence for these treatments is low and that no definite statements on the efficacy and clinical usefulness of these treatments can be made. A further Cochrane Review on the effect of manipulation and mobilisation of neck pain found low quality evidence that cervical and thoracic manipulations may provide pain reduction [18]. An additional Cochrane Review on the effect of exercises for mechanical neck disorders concluded that the summarised evidence indicates that there is a role for exercises in the treatment of acute and chronic mechanical neck pain plus headache but that the relative benefit of each type of exercise needs extensive research [19].

However, none of these reviews have covered the majority of commonly used physiotherapy modalities in one in order to get an overview of the subject. Besides, the effect of specific physiotherapy treatments in specific subgroups of chronic pain patients is an important topic which has not yet been examined. Clinicians and policy makers need evidence from research to inform and guide clinical practice and policy. Patients and researchers also need such information to support shared decisions and to set priorities for future research.

The aim of this study was to review the literature systematically and discuss the quality of evidence of commonly used physiotherapy interventions (exercise, manual therapy, and electrotherapy) aimed at improving outcomes (on pain, function, and quality of life) important for patients with chronic neck pain [20]. Neck pain was defined as pain located in the anatomical region of the neck [21]. Pain was considered chronic if it had persisted for more than three months, as defined by the International Association of the Study of Pain.

\section{Methods}

We performed a systematic review of all available randomised controlled trials on the subject of physiotherapy for neck pain to determine the effects of physiotherapy interventions on pain, function, and quality of life in neck-pain patients and to explore whether beneficial effects could be explained by biases affecting individual trials [22]. Study selection, assessment of eligibility criteria, and data extraction were carried out based on a predefined, peer-reviewed protocol according to the Cochrane Collaboration's guidelines [23]. This paper was prepared in accordance with the PRISMA statement [24].

2.1. Literature Search. We searched five bibliographic databases (MEDLINE, EMBASE, CINAHL, Cochrane Library, and PEDro) from January 1990 to January 2012 with a structured, pre-defined, search strategy [25]. The search strategy was "Neck Pain AND Physiotherapy Intervention." For neck pain, the following terms were combined with OR: "whiplash/WAD," "neck injury," "neck sprain/strain," "neck ache," "cervical sprain/strain," "cervical disorder/syndrome," "cervical spondylosis/itis," "cervical osteoarthritis", "cervicodynia", "cervicobrachial pain/disorder/syndrome", "myofascial pain/disorder/syndrome," "trapezius myalgia," "postural syndrome," and "nonspecific neck pain." For physiotherapy interventions, the following terms were combined with OR: "physiotherapy," "physical therapy," "rehabilitation," "intervention studies," "exercise," "exercise therapy," "exercise movement techniques," "manual therapy," "manipulative medicine," "mobilisation/mobilization," "musculoskeletal techniques," and "electric/electro stimulation therapy." All terms were searched as free text as well as keywords, where this was applicable. Limitations were human studies in the English, German, Dutch, Danish, Norwegian, and Swedish languages, in the time span of January 1990 to January 2012. To assure that the included studies followed scientifically sound methods and the data therefore were well documented, we set a limit for inclusion to publications from 1990 and onwards.

Reference lists of review articles and included studies were searched to identify other potentially eligible studies. An additional search was conducted via the scientific search machine http://www.scirus.com/, using the following search terms combined with AND: "chronic neck pain," "physiotherapy."

2.2. Selection Criteria. Studies were included if participants were older than 18 years of age and had chronic neck pain for more than three months (therefore considered chronic). Chronic neck pain was defined as (i) chronic whiplashassociated disorders (WAD); (ii) chronic non-specific neck pain, including work-related neck pain, myofascial neck pain, upper trapezius myalgia, chronic neck pain associated with degenerative findings with or without radicular findings, or other surrogate terms.

Eligible interventions were physiotherapy interventions commonly used in the treatment of musculoskeletal pain: (i) exercise therapy, including specific types of exercises, for example, neuromuscular training, strength training, and endurance training; (ii) manual therapy, for example, massage, manipulations, and mobilisations; (iii) electrotherapy, for example, TENS, low-level laser, or other surrogate terms. Acupuncture was not considered a physiotherapy technique since this technique is not part of physiotherapy in all countries. Comparison of the therapy had to be made with no treatment (e.g., waiting list controls), or other conservative active therapies called "care as usual," or sham therapy. Anticipating that only a limited number of trials available used placebo/sham control, we decided also to include trials in which an active control was used as a cointervention.

To be eligible for inclusion, a study must apply at least one pain measurement prior to and following the intervention, which was an outcome considered to be of major importance to the patients. Self-reported function and disability [26], self-reported quality of life [27], objective physical function, and clinical tests were considered minor outcomes and therefore not considered necessary inclusion criteria [28-30]. Only randomised controlled trials were accepted. Exclusion criteria were studies with participants with acute or subacute 


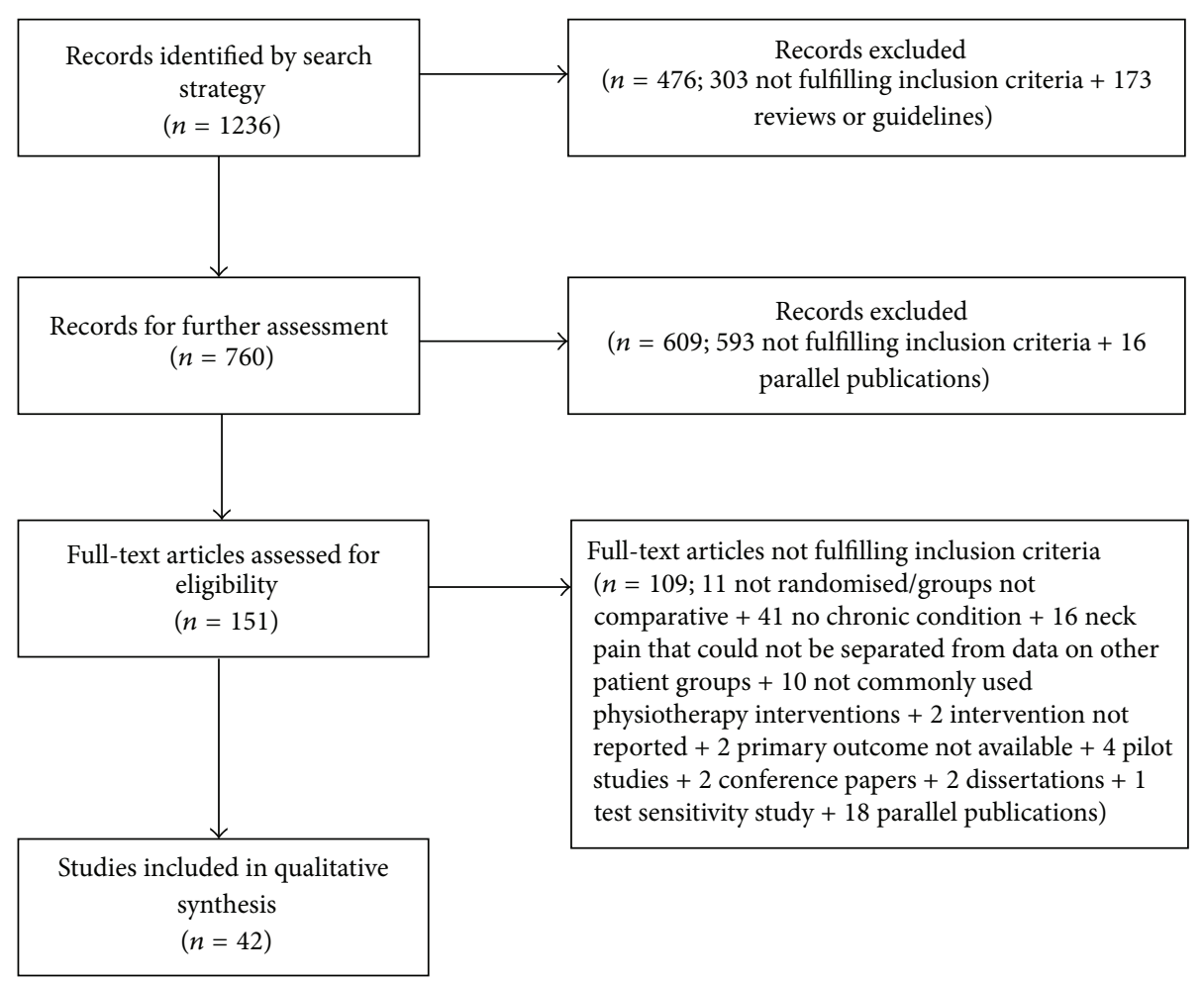

FIGURE 1: Flow diagram of the selection process of included studies.

neck pain, neck pain with definite or possible long tract signs, neck pain due to specific pathological conditions (e.g., fractures, tumours, infections, inflammatory processes, ankylosing spondylitis, and rheumatoid arthritis), and headache.

We created a reliable process through consequently two reviewers who independently conducted the study selection and assessment of eligibility criteria. Similarly, two reviewers independently conducted data abstraction and assessed the risk of bias. Disagreements were resolved through consensus with a third reviewer being consulted if there was disagreement.

2.3. Data Extraction and Evidence Synthesis. Data regarding publication status, trial design, patient characteristics, treatment regimens, outcome methods, results, and funding were extracted on a standardised form using a custom-made Microsoft Excel spreadsheet.

We assessed the risk of bias by using the Cochrane Collaboration's tool for assessing risk of bias as presented in [23]. Each of the following domains would be considered adequate - that is, presumably with a low risk of bias (i) "adequate sequence generation"; (ii) "allocation concealment"; (iii) "blinding"; (iv) "incomplete outcome data addressed"; (v) "free of selective outcome reporting"; (vi) "free of other bias (i.e., whether a study sponsor would benefit economically from a positive outcome). Each of these key components of methodological quality was assessed on an Adequate/Unclear/Inadequate basis. We used The Cochrane Collaboration's approach for summary assessments of the risk of bias for each important outcome across domains within a trial [23].
Due to the limited number of studies investigating each of the specific interventions, it was decided that both metaanalytical and level of evidence approaches would be inappropriate. Therefore, a narrative approach where we evaluated the study and results between groups within a trial was used to summarise the findings. To formulate conclusions, only results from trials at low risk were considered as evidence for an intervention.

\section{Results}

The literature search identified 4921 relevant studies (1110 from EMBASE, 1568 from MEDLINE, 1239 from CINAHL, and 491 from PEDro), of which 3685 were duplicates, leaving 1236 potentially eligible studies to be screened (see Figure 1). Following screening of titles and abstracts, 151 potentially relevant studies were identified and retrieved in full text. Finally, 42 randomised controlled trials, involving 3919 patients, fulfilled the selection criteria and were considered suitable for inclusion. The selection process and reasons for exclusions are presented in Figure 1.

3.1. Study Characteristics. Study characteristics and study results are presented under the categories exercise therapy (25 trials, 18 regarding chronic non-specific neck pain, and seven regarding chronic neck pain related to whiplash); manual therapy (six trials, all related to chronic non-specific neck pain); and electrotherapy (11 trials, all related to chronic nonspecific neck pain) in Appendix A, Tables 1-4. 
The trials covered the following intervention topics: (i) exercise therapy: various types of dynamic and isometric exercises, general aerobic exercises, exercises with a focus on strength, endurance, proprioception and coordination, specific neck stabilising exercises, craniocervical-flexion exercises, posture, behavioural graded activity, relaxation, body awareness, myo-feedback training, and multimodal physiotherapy; (ii) manual therapy: massage, manipulation, and traction; (iii) electrotherapy: laser, transcutaneous nerve stimulation (TENS), ultrasound, and repetitive magnetic stimulation (rMS).

Sham therapy or waiting list controls were used as control groups in 12 trials; 10 trials used a control group consisting of a self-management book, health-counselling, or other interventions, clearly distinguished from the active intervention group; six trials used active-treatment control reported as "treatment as usual"; active-treatment control was used in 14 trials.

Primary outcome measures were self-reported pain and/or self-reported pain and disability in 41 trials; when primary outcome measures were not reported, all outcome measures were considered. One trial had an objective test as primary outcome, yet pain was included in the secondary outcome measures.

3.2. Risk of Bias. Risk of bias is presented in Appendix B, Table 5.

Overall, the quality of reporting on methodological issues varied. Table 5 shows the judgements ("Adequate," "Unclear," and "Inadequate") for each of the domains. As can be seen, 28 of 42 trials succeeded in reporting on adequate sequence generation; 18 trials described adequate allocation concealment; four trials adequately reported on attempts to blind participants, personnel, and outcome assessors; 22 trials adequately reported on missing outcome data, using intention-to-treat analysis; three trials adequately reported on selective outcome reporting by referring to a published and available protocol for comparisons; and 25 trials adequately reported on funding and the role of funding.

The summary assessment of risk of bias revealed 19 trials at low risk of bias $[34-39,45,47-49,51,55-60,63,64]$ and 23 trials as unclear or at high risk of bias [31-33, 40-44, 46, 50, $52-54,61,62,65-72]$, and for this reason their results were not considered as evidence. Of these 19 trials at low risk of bias, 11 trials found no difference between intervention groups [34, $36-39,45,48,49,56,58,60]$, and eight trials found an effect on pain of the intervention $[35,47,51,55,57,59,63,64]$.

All studies are described in detail in Appendix A, Tables 1-4. All trials at low risk of bias, showing an effect on pain, are, furthermore, presented in the following section. According to the described criteria, the evidence for each intervention will following be summarised at the end of each section.

\subsection{Effect of Physiotherapy Interventions}

\subsubsection{Exercise}

Effect of Exercise on Pain in Patients with Chronic Nonspecific Neck Pain. As shown in Appendix A, Table 1, 18 trials examined the effect of various types of exercise in patients with chronic neck pain; nine of these were at unclear or high risk of bias [31-33, 40-44, 46], and nine were at low risk of bias [34-39, 45, 47, 48]. Seven of the trials at low risk examined the effect of different types of exercise, including proprioception exercises (eye-head coordination), craniocervical flexion exercises (C-CF), neck stabilisation exercises, stretching, strengthening, and behavioural graded activity programme, but did not find statistically significant difference on pain between groups following intervention $[34,36-39,45,48]$. Two of the trials at low risk of bias succeeded in finding an effect on pain from the intervention, and for this reason, their results were considered evidence of use of exercise.

(1) Gustavsson et al. [35] examined a multicomponent pain and stress self-management group intervention (PASS) versus a control group receiving individually administered physiotherapy (IAPT). There was a statistically significant effect on ability to control pain $(P<0.001)$ and on neck-related disability (NDI) $(P<$ 0.001 ) in favour of PASS at the 20-week followup.

(2) Ylinen et al. [47] examined three interventions: intensive isometric strength training versus lighter endurance training versus a control group. The two training groups had an additional 12-day institutional rehabilitation programme. At the 12-month followup, both neck pain and disability had decreased in both training groups compared with the control group $(P<0.01)$.

No trials with low risk of bias supported single use of proprioception exercises (eye-head co-ordination), craniocervical flexion exercises (C-CF), or neck stabilisation exercises for pain. No trials with low risk of bias support the use of stretching.

Effect of Exercise on Pain in Patients with Chronic WhiplashAssociated Disorder. As shown in Appendix A, Table 2, seven trials examined the effect of various types of exercise in patients with chronic WAD; three of these were at low risk of bias $[49,51,55]$, and four were at unclear or high risk of bias [50, 52-54]. One of the trials at low risk of bias examined the effect of adding biofeedback training to a rehabilitation programme, but found no difference in effect between groups [49]. Two trials at low risk of bias succeeded in finding an effect on pain from the intervention, and for this reason, their results were considered evidence of use of exercise.

(1) Jull et al. [51] examined a multimodal physiotherapy programme (including exercises, education, and ergonomics) versus a self-management programme. The multimodal physiotherapy programme group attained a statistically significant greater reduction in reported neck pain and disability (NDI) posttreatment $(P=0.04)$.

(2) Stewart et al. [55] examined exercise (e.g., endurance, strength, aerobic, coordination, and cognitive behavioural therapy) versus advice alone. Exercise and advice were more effective than advice alone at 


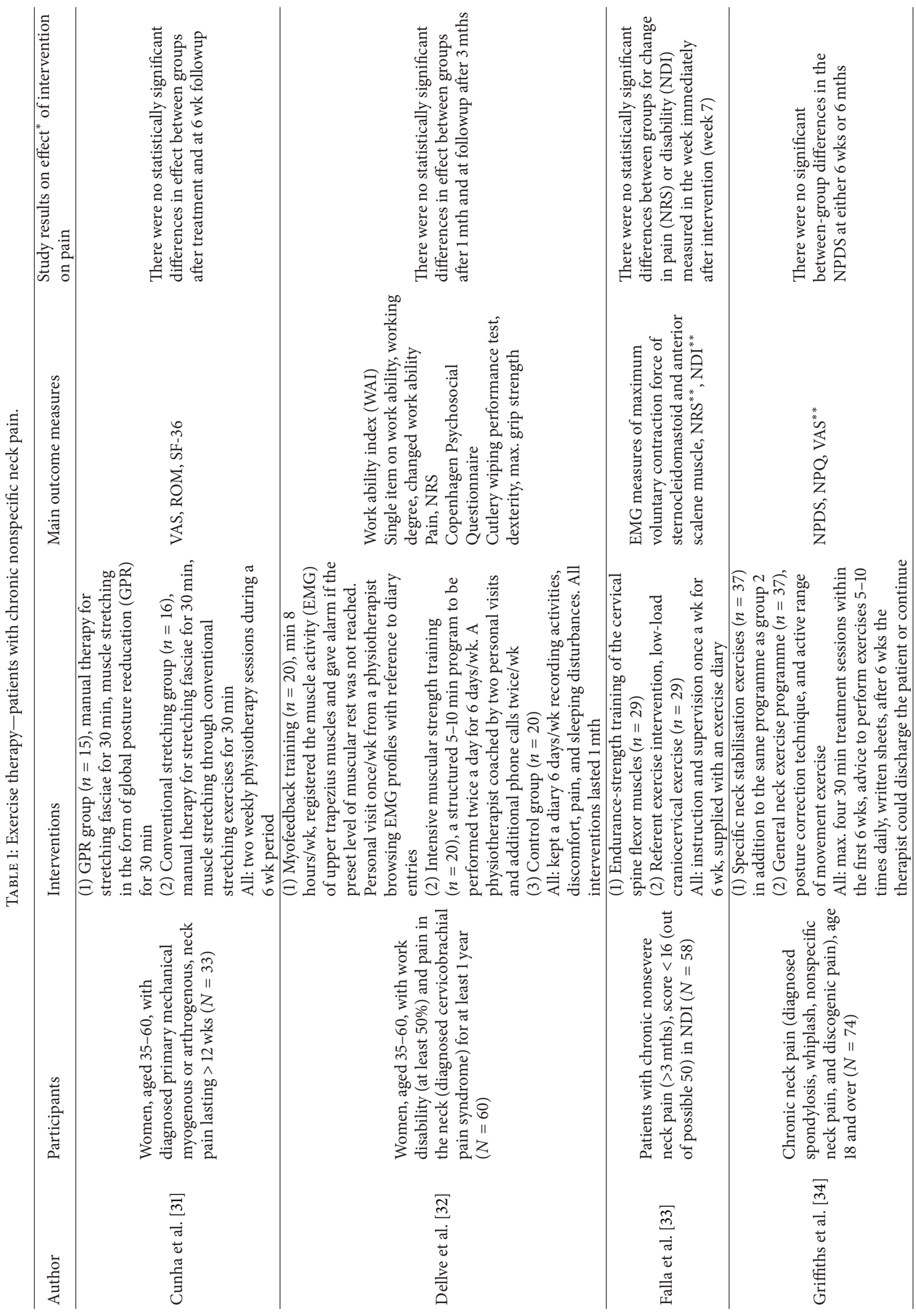




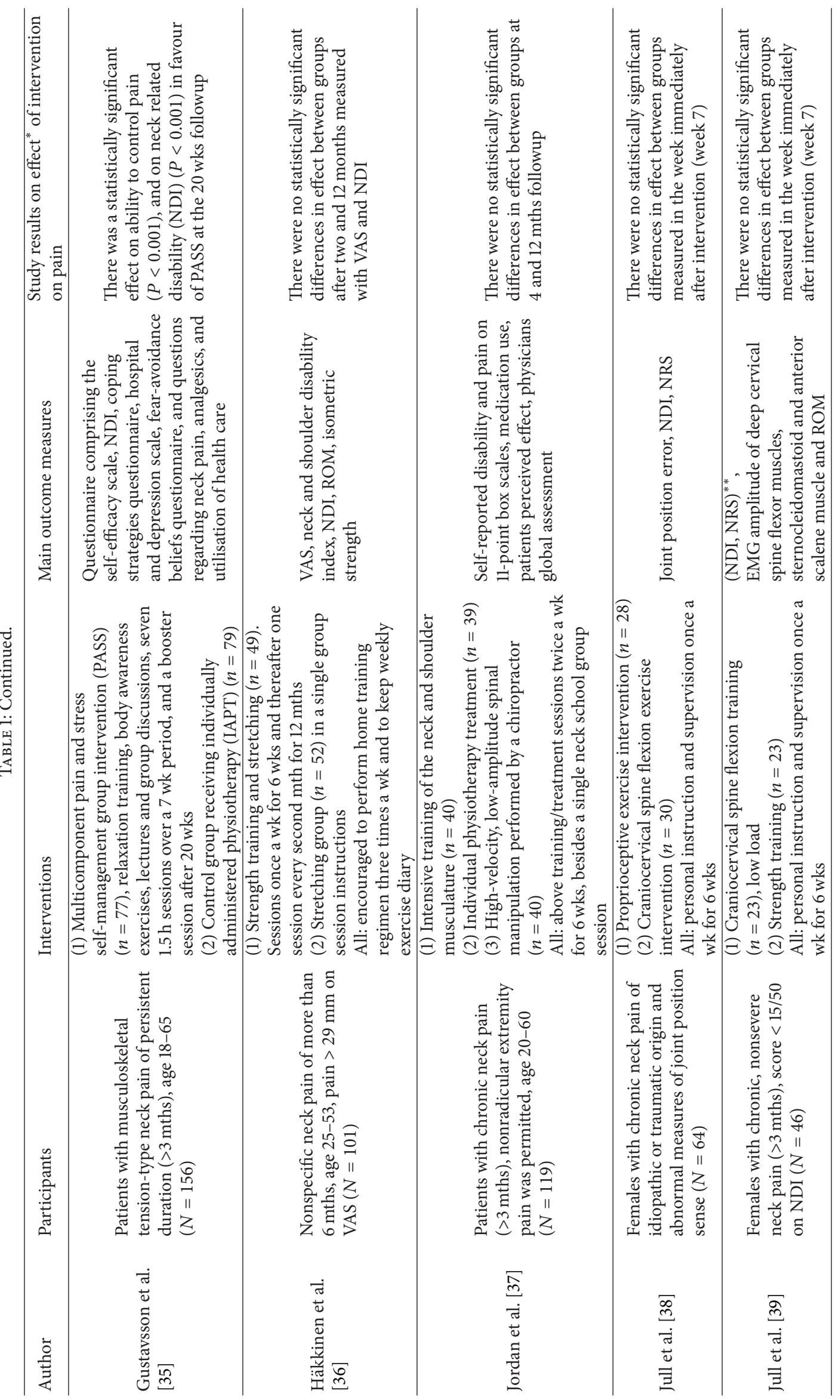




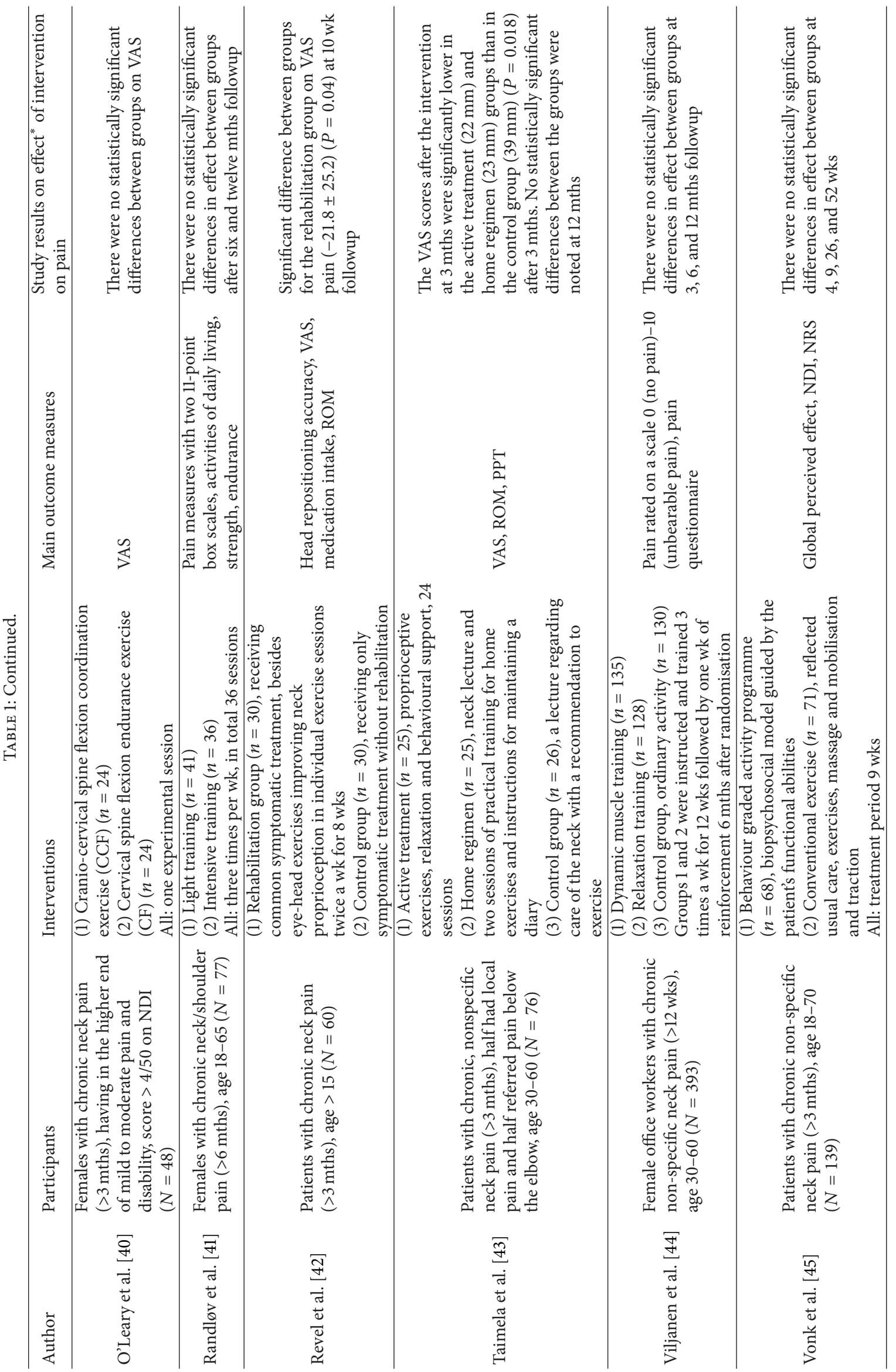




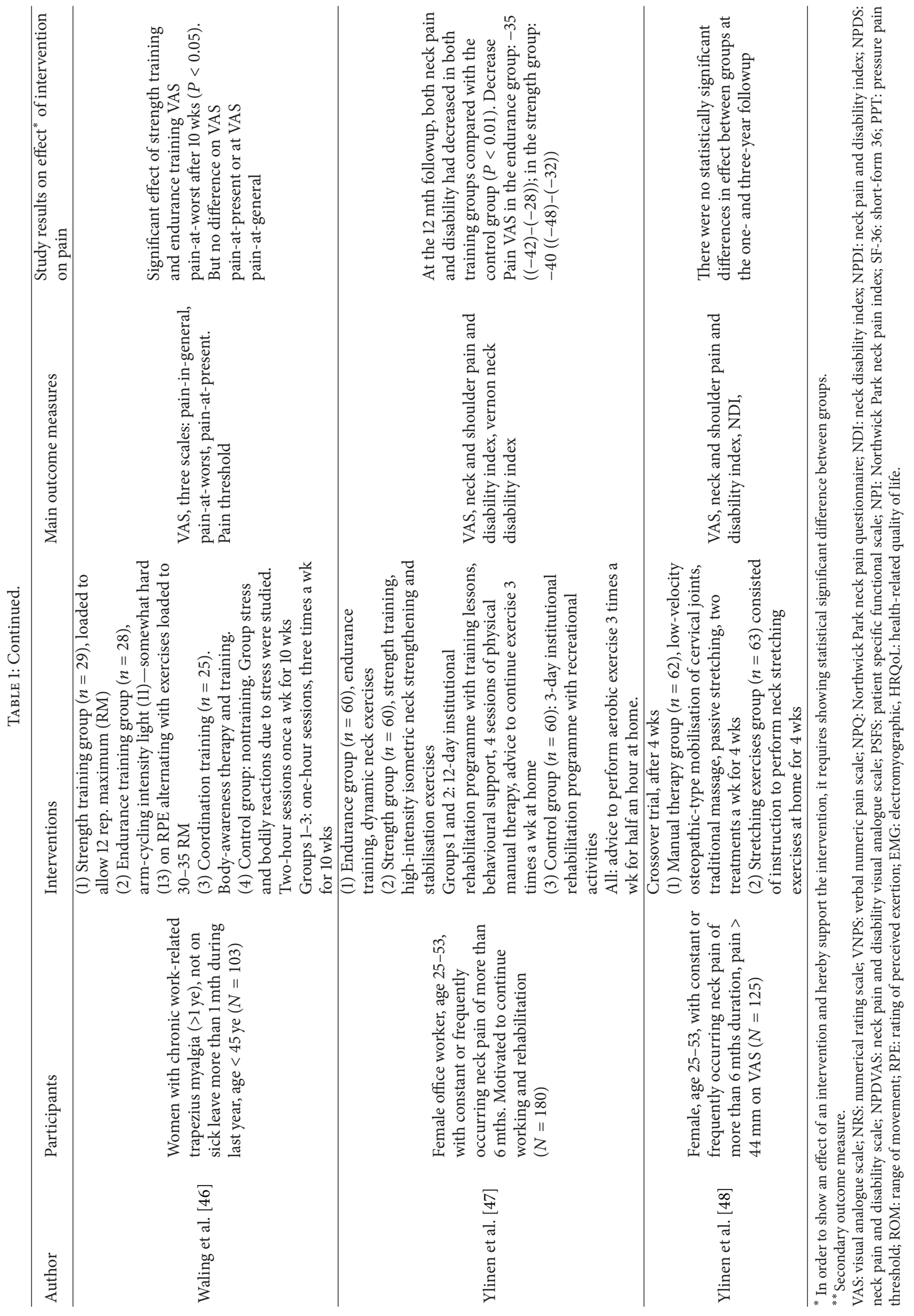




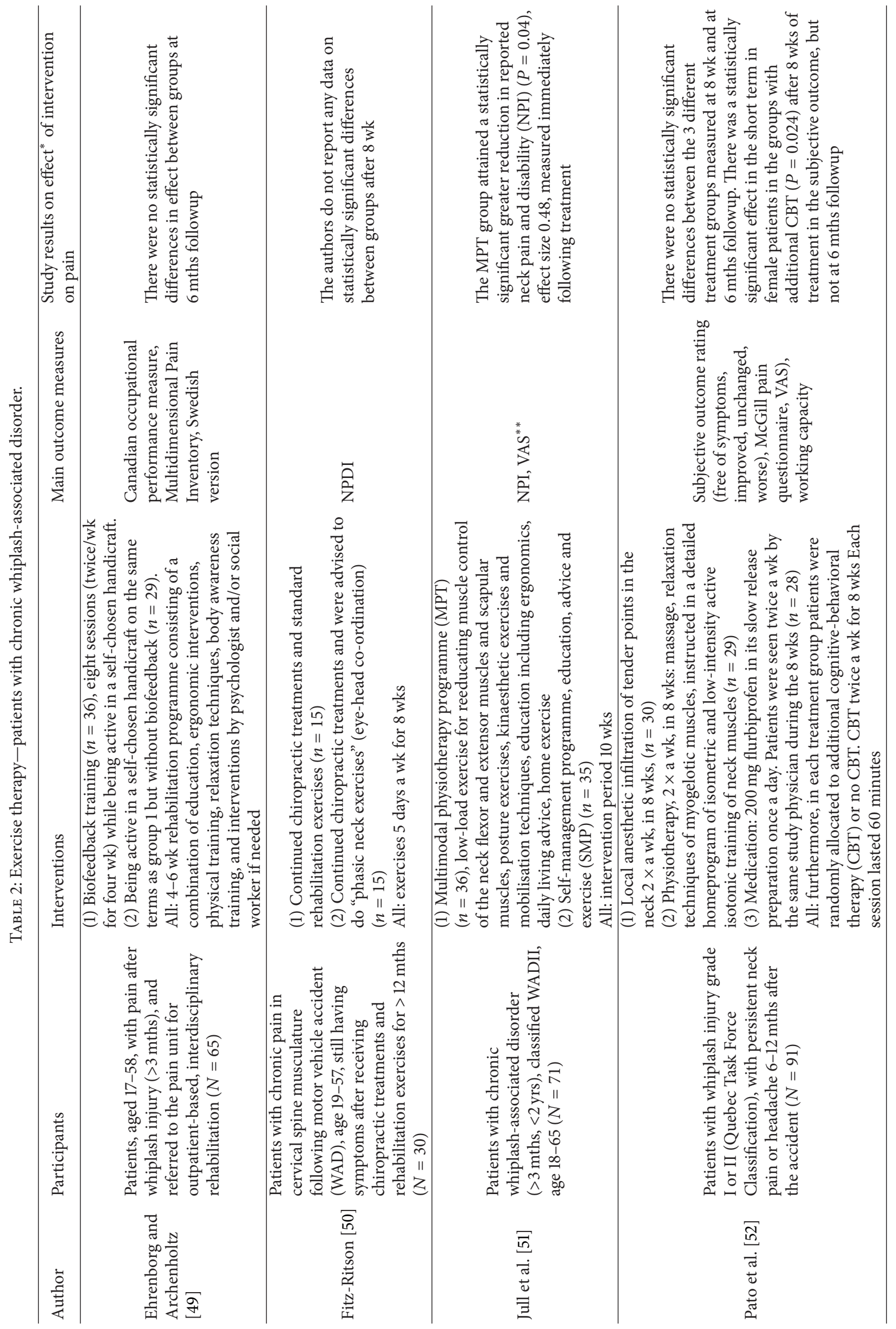




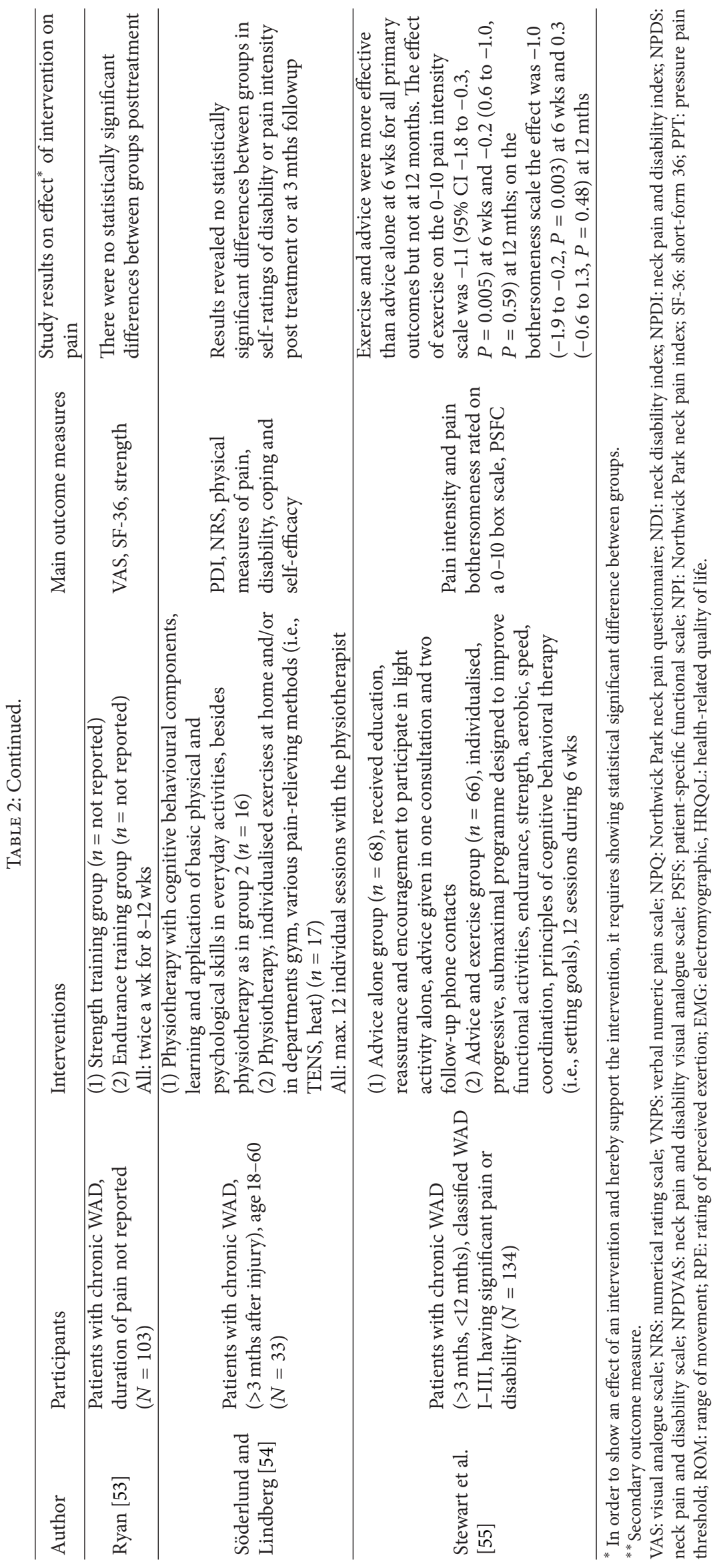




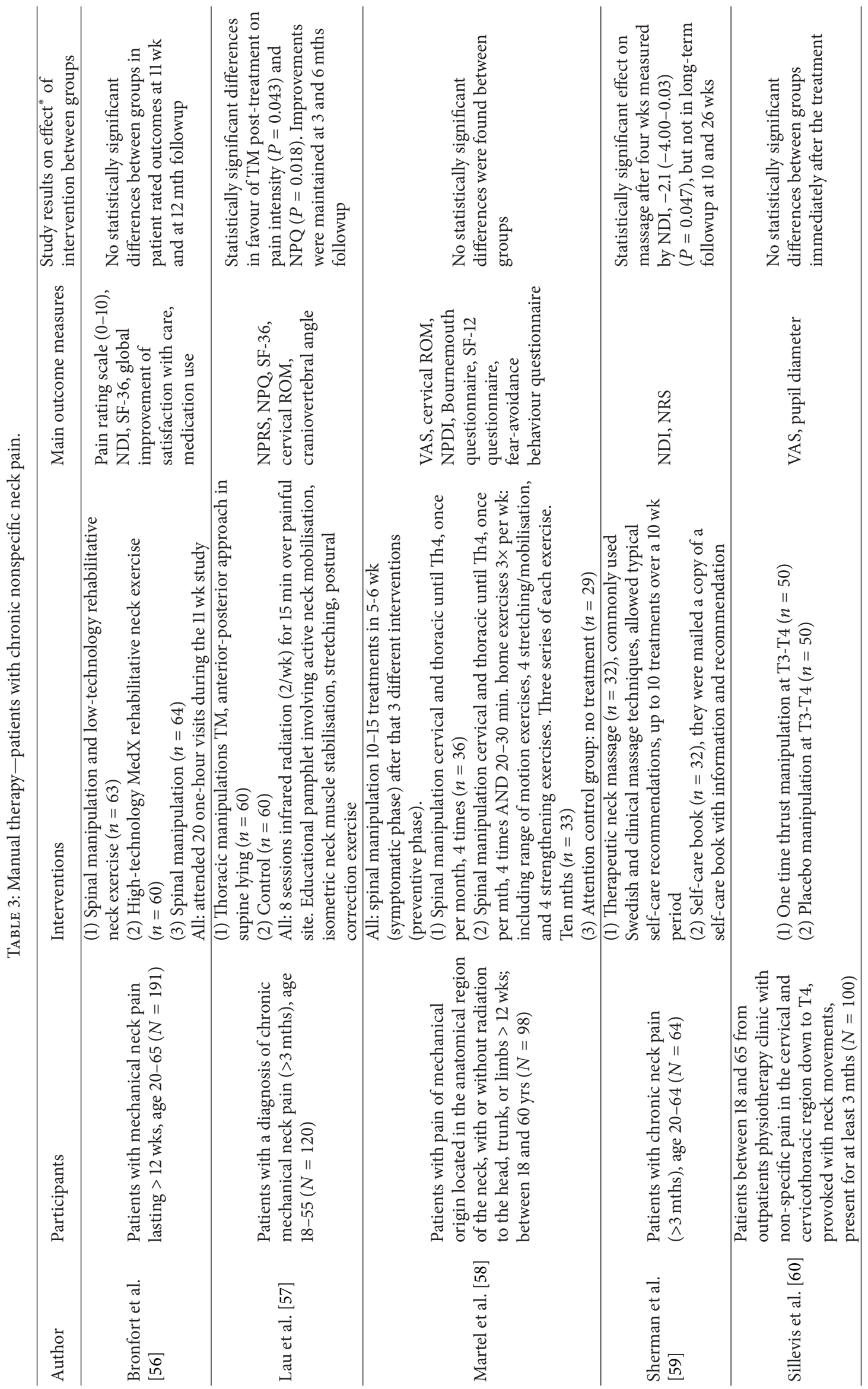




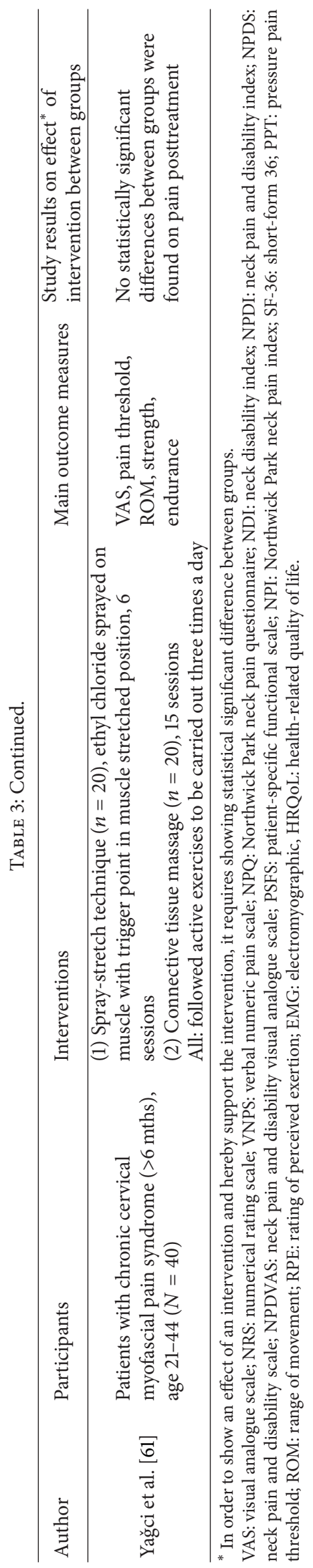




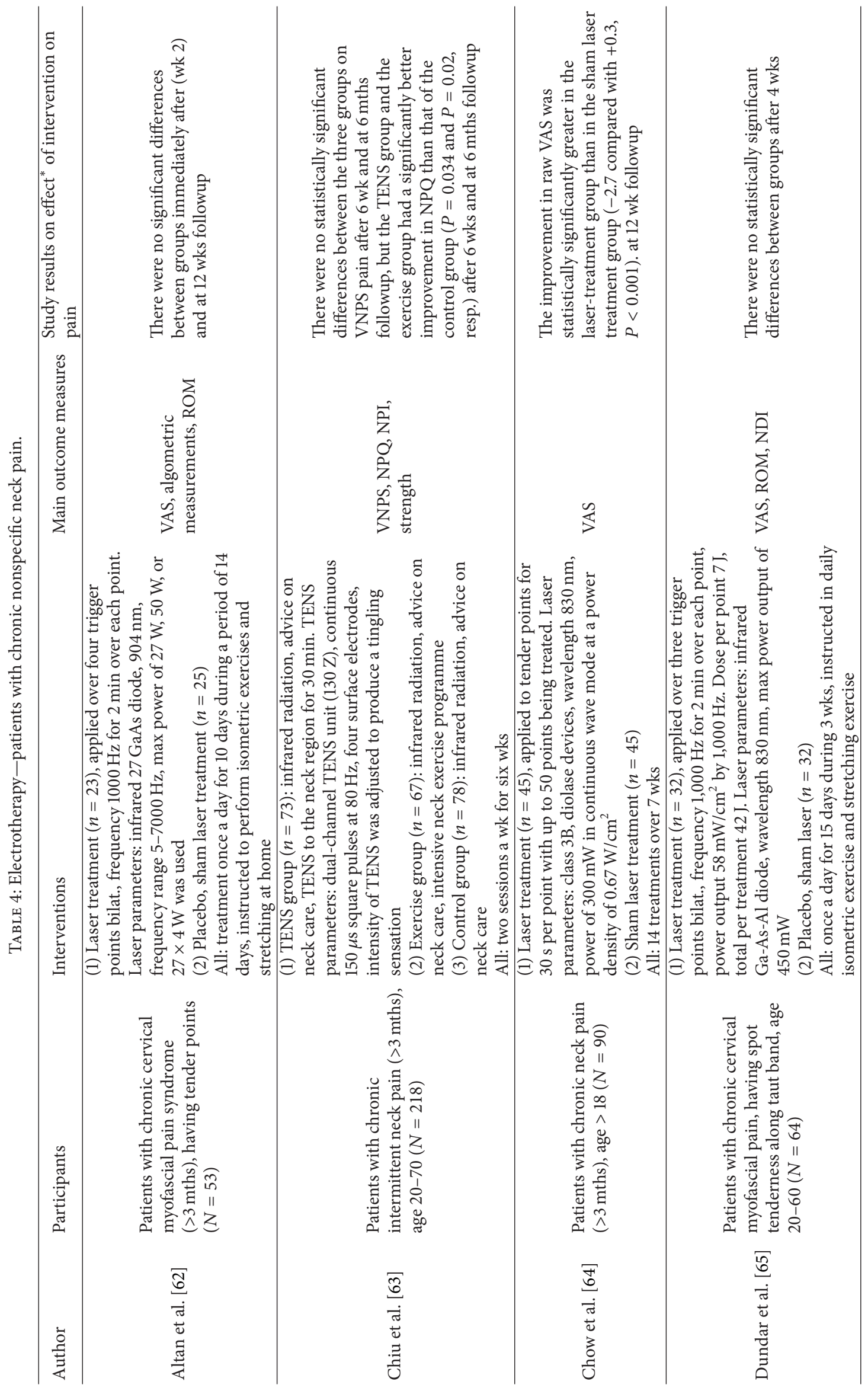




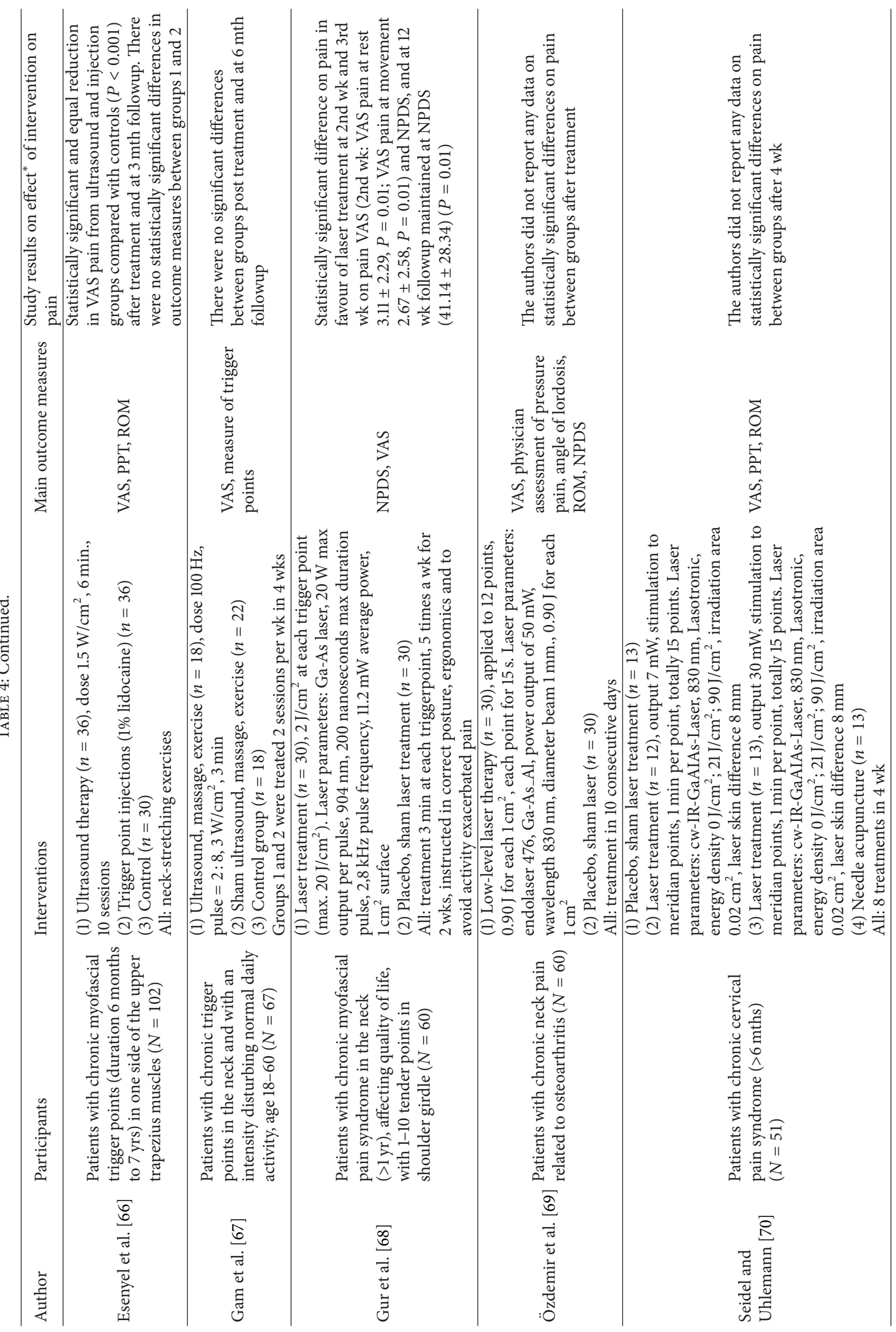




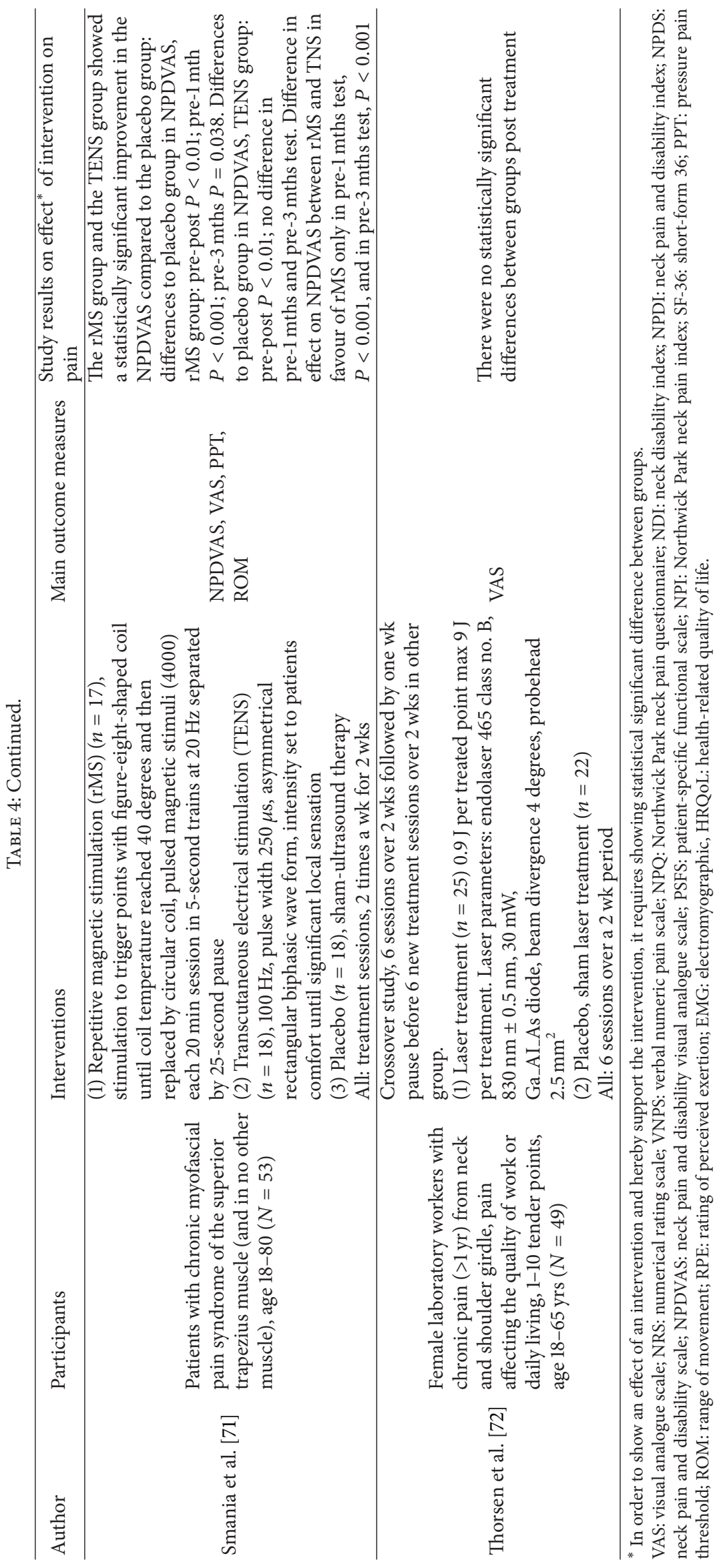




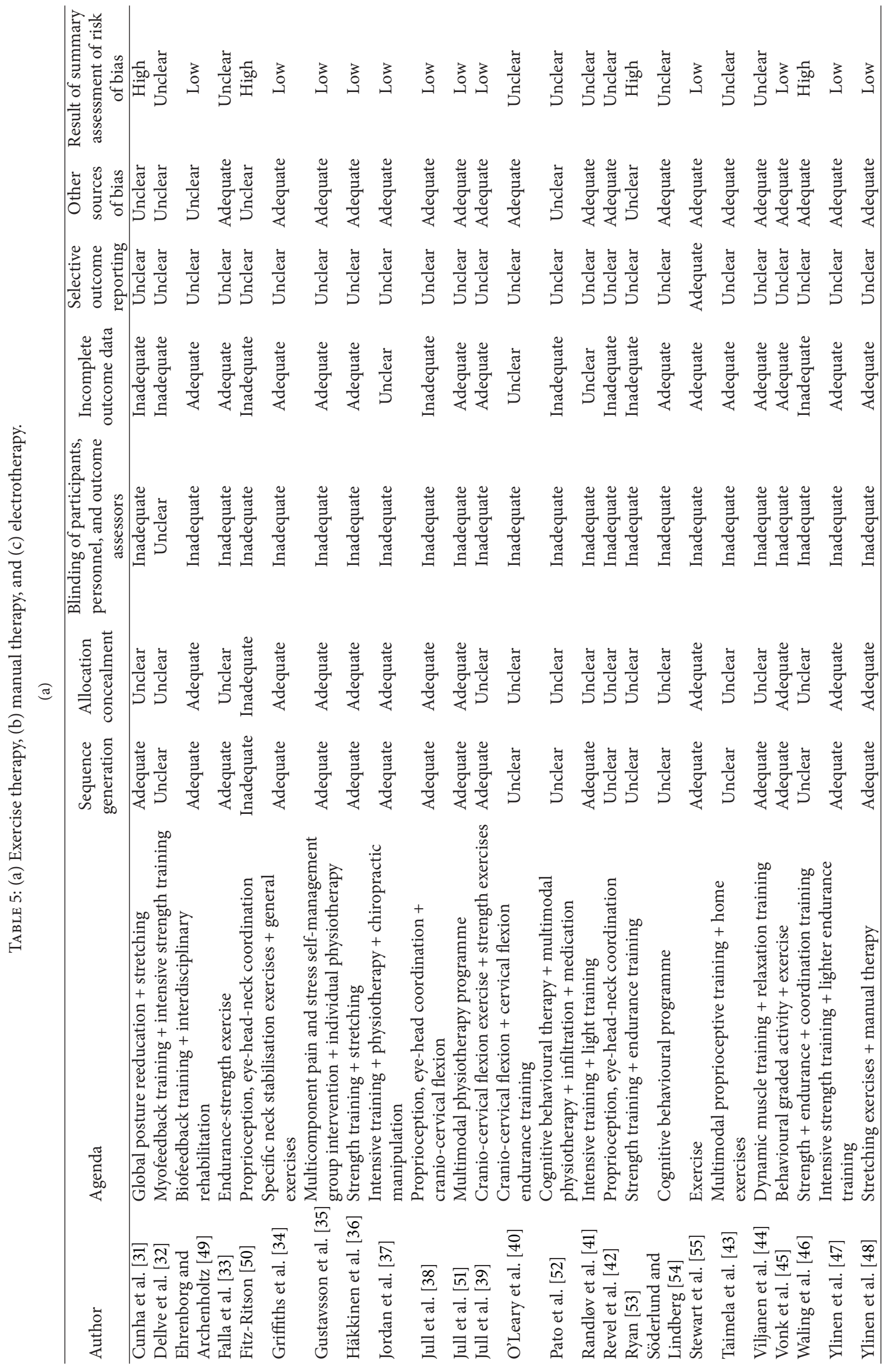




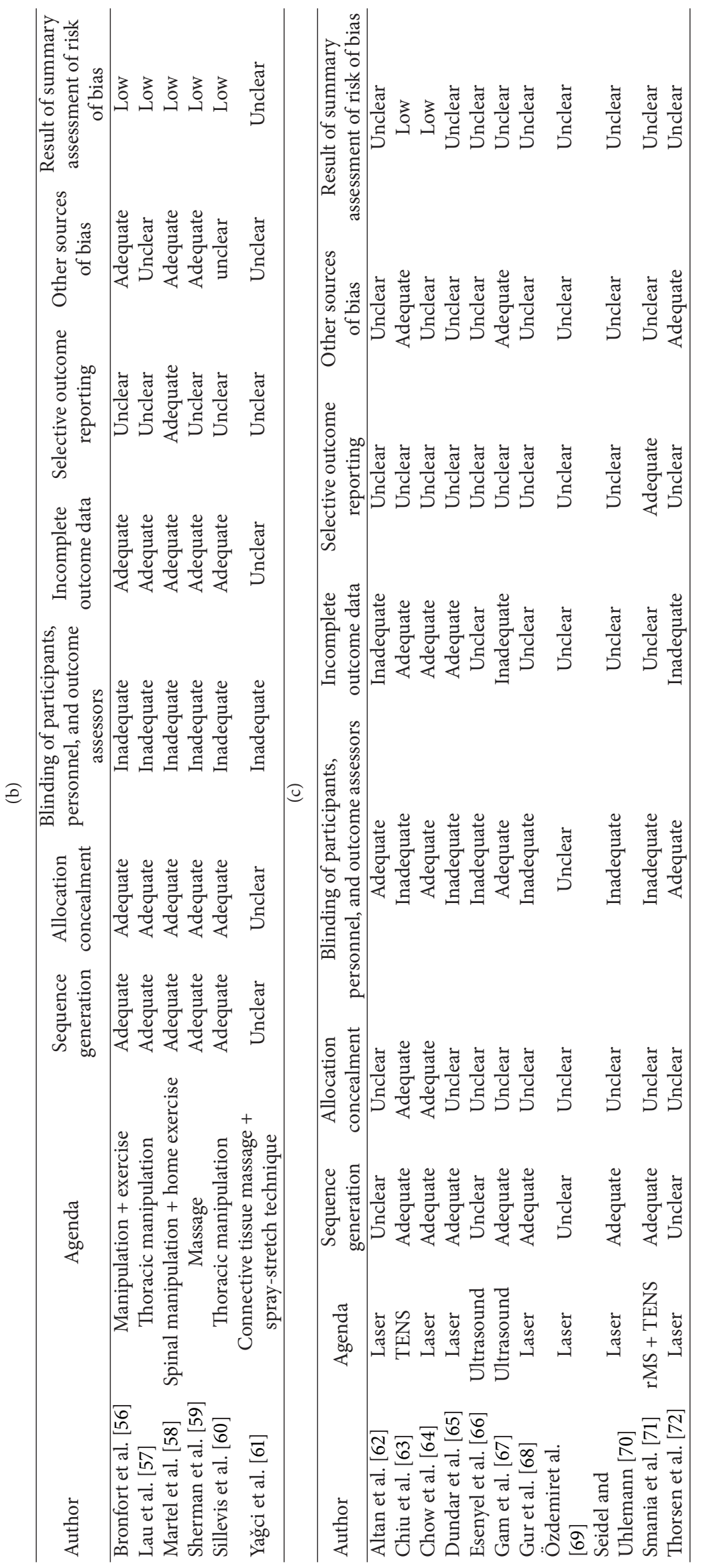


6 weeks on pain intensity scale $(P=0.005)$ and on a bothersomeness scale at 6 weeks $(P=0.003)$ and at 12 months $(P=0.003)$.

No trials at low risk of bias support the use of EMG biofeedback.

\subsubsection{Manual Therapy}

Effect of Manual Therapy on Pain in Patients with Chronic Nonspecific Neck Pain. As shown in Appendix A, Table 3, six trials examined the effect of various types of manual therapy in patients with chronic non-specific neck pain [5661]. One of the trials was at unclear risk of bias, and for that reason not considered evidence [61]. Three trials at low risk of bias examining the effect of spinal manipulations found no difference between groups $[56,58,60]$. Two trials succeeded in finding an effect on pain from the intervention. Both trials had a low risk of bias, and for this reason, their results were considered evidence of use of manual therapy.

(1) Lau et al. [57] examined thoracic manipulation versus a control group. They found statistically significant differences in favour of thoracic manipulation posttreatment on pain intensity $(P=0,043)$ and pain and disability (NPQ) $(P=0,018)$. Improvements were maintained at 3, and 6-month followup.

(2) Sherman et al. [59] examined massage versus a selfcare book. They found statistically significant effect on massage following four weeks of treatment on neck pain and disability (NDI) $(P=0.047)$, but not at longterm followup at 10 and 26 weeks.

No trials at low risk of bias support the use of traction.

\subsubsection{Electrotherapy}

Effect of Electrotherapy on Pain in Patients with Chronic Nonspecific Neck Pain. As shown in Appendix A, Table 4, 11 trials examined the effect of various types of electrotherapy in patients with chronic non-specific neck pain; two of these were at low risk of bias $[63,64]$, and nine were at unclear or high risk of bias $[62,65-72]$. The two trials at low risk of bias both succeeded in demonstrating an effect on pain from this type of intervention and for this reason; their results were considered evidence of use of electrotherapy.

(1) Chiu et al. [63] examined three interventions: TENS versus exercise versus a control group. There were no statistically significant differences between the three groups on pain (VNPS) after 6-week and at 6-month followup, but the TENS group and the exercise group had a significantly better improvement in neck pain and disability (NPQ) than the control group $(P=$ 0.034 and $P=0.02$, resp.) after 6-week, and at 6month followup.

(2) Chow et al. [64] examined laser versus sham laser treatment. The improvement in VAS was statistically significantly greater in the laser treatment group than in the sham laser treatment group $(-2.7$ compared with $+0.3, P<0.001)$ at 12 -week followup.

No trials at low risk of bias support the use of ultrasound therapy. No trials at low risk of bias support the use of rMS.

\section{Discussion}

4.1. General Interpretation. In this review, we assessed the effect of various interventions for the treatment of chronic neck pain and evaluated the methodological quality of the trials. Our findings emphasise the importance of taking the risk of bias into consideration when evaluating the evidence of an intervention.

Trials varied substantially regarding their internal validity, although the methodological quality of the RCTs in general appeared to be somewhat low with an unclear or high risk of bias. We identified various methodological flaws that may have implications for the internal validity of the trials and consequently may result in biased outcomes. Key domains in this context were randomisation, blinding, and incomplete outcome data.

Our evaluation also exposes a widespread use of withingroup analyses, claiming statistically nonsignificant results to be beneficial. Results were frequently analysed and reported as if they were uncontrolled within-group studies, which consequently led to misinterpretation of results. To some extent this may be due to the absence of a control group in many trials, and the use of an active treatment as a comparative group makes the "proof" of a truly statistically significant effect more difficult to find. We believe that attention should be paid to inadequate interpretation of a trial result when authors inadequately interpret lack of difference in terms of efficacy [73-76].

4.2. Effect of Physiotherapy on Chronic Neck Pain. Overall, the evidence of effect of physiotherapy for chronic neck pain is strengthened. Yet, for some of the treatments offered, no definite effect and clinical usefulness can be shown. This does not necessarily implicate that these treatments have no effect, only that the present evidence is not sufficient.

Physiotherapy interventions for chronic neck pain showing the strongest support for an effect on pain are strength and endurance training (supported by two trials by Stewart et al. [55] and by Ylinen et al. [47], treating patients with chronic WAD and patients with chronic non-specific neck pain, resp.). In patients with chronic WAD, multimodal physiotherapy was also shown to have a beneficial effect by one trial by Jull et al. [51]. In patients with chronic non-specific neck pain, the use of cognitive/behavioural components in exercise was supported by one trial by Gustavsson et al. [35]. In regard to manual therapy, massage seems to have an effect on pain in patients with chronic non-specific neck pain, supported by one trial by Sherman et al. [59], and thoracic manipulation seems to have an effect on pain, supported by one trial by Lau et al. [57]. Within the area of electrotherapy, both laser therapy and TNS seem to have an effect on pain in patients with chronic non-specific neck pain. This was supported by one trial by Chow et al. [64] and one trial 
by Chiu et al. [63]. No trials supported the isolated use of proprioception (eye-head co-ordination), cranio-cervical flexion training, stretching, ultrasound therapy, rMS, and traction.

When looking deeper into the actual components of the various interventions in the above-mentioned trials, four of them-despite the differences, diversity, and individual features of the interventions-seem to have several characteristics in common: The interventions can be considered to be rehabilitative interventions of multimodal physiotherapy with a focus on exercise, including cognitive-behavioural components. This is based on (1) the trial by Stewart et al. [55] showing effect of mixed exercises, where the intervention besides submaximal training, stretching, and aerobic endurance included coordination programme designed to improve functional activities and principles of cognitive behavioural therapy (i.e., setting goals); (2) the trial by Ylinen et al. [47] showing effect of strength training and endurance training, where training groups had an additional 12-day institutional rehabilitation programme with training lessons, behavioural support, ergonomics, sessions of physical manual therapy-including massage/mobilisations_-and advice to continue exercise; (3) the trial by Jull et al. [51] showing effect of a multimodal physiotherapy, including low-load exercise for reeducating muscle control of the neck flexor and extensor muscles and scapular muscles, posture exercises, kinaesthetic exercises, and mobilisation techniques, and in addition education including ergonomics, daily living advice, and home exercise; (4) finally, the trial by Gustavsson et al. [35] who found effect from a multi-component pain and stress self-management group intervention-including relaxation training, body awareness exercises, and lectures and group discussions-regarding coping with pain in terms of patients' self-reported pain control, self-efficacy, and disability. Our main results are consistent with findings of previous reviews of interventions for neck pain. The Cochrane Review by Kay et al. [19] on the effect of exercises for mechanical neck disorders concluded that the summarised evidence indicates that there is a role for exercises in the treatment of acute and chronic mechanical neck pain plus headache, but that the relative benefit of each type of exercise needs extensive research. Our review on chronic neck pain agrees with the present conclusion regarding exercise, yet our findings tend to favour strength and endurance training, as well as multimodal physiotherapy in addition to pain and stress selfmanagement. The superior effect of strength training and endurance training may be due to the physical impairments found in the chronic condition [77-80].

Our review adds new knowledge regarding the evidence for use of massage. Our findings are in discrepancy to a Cochrane Review by Haraldsson et al. [13] who concluded that the effectiveness of massage for improving neck pain and function remains. Yet the quoted review was last updated in 2004, and the trial by Sherman et al. [59] supporting massage was published in 2009. Our findings on the evidence of manipulation are in line with another Cochrane Review by Gross et al. [18] on the effect of manipulation and mobilisation for neck pain, who found low quality evidence that cervi$\mathrm{cal}$ and thoracic manipulations may provide pain reduction.
We too found evidence that thoracic manipulations may have an effect on pain [57]. Regarding low-level laser therapy, our findings are consistent with the findings of a review by Chow et al. [81], who found that low-level laser therapy reduces pain in patients with chronic neck pain. A Cochrane Review from 2007 [17] on the effect of conservative treatment for whiplash concluded that clearly effective treatments are not found for treatment of acute, subacute, or chronic symptoms. The findings of our newer review do support multimodal physiotherapy and mixed exercise programmes for chronic WAD. The explanation for the difference may be that the Cochrane Review by Verhagen in 2007 was not updated after January 2007, and our findings are based on more recent research, namely, two trials published later in 2007 [51, 55]. A more recent review by Teasell et al. [82] found evidence to suggest that exercise programmes are the most effective noninvasive treatments for patients with chronic WAD. Our findings give support to the use of cognitive-behavioural element, and to pain and stress self-management. This is in discrepancy to another Cochrane Review by Gross et al. [14] on patient education for neck pain, concluding that there is no strong evidence for the effectiveness of educational interventions in various neck disorders. This difference may be due to the use of only single-modal trials in their review rather than multimodal trials as used in the current review.

4.3. Strengths and Weaknesses of Review Procedures. To our knowledge, this is the first systematic review on interventions for chronic neck pain addressing the majority of commonly used physiotherapeutic modalities in one study, in order to get an overview of the subject area.

The search strategy and selection criteria we used were quite strict and easy to apply and according to normal procedures for conducting systematic reviews [23]. Yet the following limitations of the literature search may have introduced a bias: some relevant trials may have been missed if they used other keywords, although this is not very likely. We had limitations in language, and this may have led to missing studies from countries in Eastern Europe with a tradition of physiotherapy research, like Poland. We decided to limit our search from 1990 to January 2012. This was due to physiotherapists prior to this time not being trained in scientific methods and also that RCTs were rare. Studies earlier than 1990 would in general not be following a strict protocol like the ones used for RCTs, but at best be longitudinal cohort studies.

The quality assessment was presented in a reproducible manner. However, the results may be affected by our emphases during filtering methods for synthesis evidence. We might have chosen to exclude all trials with insufficient reporting on allocation sequence and allocation concealment. However, we chose not to, since this would have left us with very few trials to assess. We assessed risk of bias, requiring a convincing mechanism to be described in order for a trial to be classified as "adequate." Our approach to this problem was to assume that the quality was inadequate unless information on the contrary was provided, and in doing so, we might have misclassified well-conducted but badly reported trials. 
The present review succeeded in a subgroup assessment of physiotherapy treatment for chronic non-specific neck pain and for chronic WAD. Yet the first group was very wide due to the mixed conditions in the group of participants. The various interventions were considered to be complex, multifaceted, and with various cointerventions, and by classifying them into intervention groups according to-what we believed to be-the trial's agenda, we may have misclassified some. On the other hand, the often used combined therapies also highlight a fundamental problem when assessing effect of specific and single physiotherapy modalities. Another issue is the quality of the intervention since the interventions were administered in different ways and in different settings. It is reasonable to expect that the way in which they were administered including the dose-response relationship could have influenced the outcome. It would have been interesting and very relevant to examine this. Herbert and Bo [83] emphasise that researchers carrying out systematic reviews should routinely examine the quality of interventions.

4.4. Future Directions. We need to know which patients will benefit from which intervention, built on well-conducted and well-reported trials, considering subgroups of patients with chronic neck pain, in order to support recommended evidence-based decisions and to set priorities for future research. We also request future trial investigators to consider to what extent cointerventions are valuable, in addition to possible confounders. Another issue to consider is the extent to which the control groups ought to be given care and attention to the same extent as the intervention groups.

\section{Appendices}

\section{A. Study Characteristics and Study Results}

See Tables 1-4.

\section{B. Risk of Bias}

See Table 5.

\section{Conflict of Interests}

The authors report no conflict of interests.

\section{Authors' Contribution}

P. Damgaard contributed to the conception, design, and writing of the study protocol and the design of search strategies; she located and obtained trial reports, helped to select and assess trials, conducted the data analysis, and drafted and approved the final paper. E. M. Bartels contributed to the conception of the study protocol and the design of search strategies; she helped to locate and obtain trial reports, and revised and approved the final paper. I. Ris helped to select and assess trials, contributed to the data analysis, and revised and approved the final paper. R. Christensen contributed to the conception, design, and writing of the study protocol, conducted data analysis, and revised and approved the final paper. All authors acted as guarantors for the paper. B. JuulKristensen contributed to the conception, design, and writing of the study protocol and the design of search strategies; she helped to select and assess trials and revised and approved the final paper.

\section{Disclosure}

The funding organisations had no role in any aspect of the study, the paper, or the decision to publish.

\section{Acknowledgment}

The authors have completed ICMJE's the Unified Competing Interest form at (available on request from the corresponding author) and want to acknowledge. The financial support for the submitted work by grants from The Danish Association for Physiotherapists, the Research Fund of the Region of Southern Denmark, the patient organization PTU-Danish Society of Polio and Accident Victims and from The Research Unit for Musculoskeletal Function and Physiotherapy at The University of Southern Denmark, and the Parker Institute, Musculoskeletal Statistics Unit, which is supported by grants from The Oak Foundation, The Danish Rheumatism Association, and Frederiksberg Hospital. The authors also declare. No financial relationships with commercial entities that might have an interest in the submitted work; no spouses, partners, or children with relationships with commercial entities that might have an interest in the submitted work; and no nonfinancial interests that may be relevant to the submitted work.

\section{References}

[1] N. Becker, A. B. Thomsen, A. K. Olsen, P. Sjøgren, P. Bech, and J. Eriksen, "Pain epidemiology and health related quality of life in chronic non-malignant pain patients referred to a Danish multidisciplinary pain center," Pain, vol. 73, no. 3, pp. 393-400, 1997.

[2] J. A. J. Borghouts, B. W. Koes, H. Vondeling, and L. M. Bouter, "Cost-of-illness of neck pain in The Netherlands in 1996," Pain, vol. 80, no. 3, pp. 629-636, 1999.

[3] P. M. Brooks, "The burden of musculoskeletal disease-a global perspective," Clinical Rheumatology, vol. 25, no. 6, pp. 778-781, 2006.

[4] M. Kjoller, K. Juel, and F. Kamper-Jorgensen, Folkesundhedsrapporten Danmark 2007, Statens Institut for Folkesundhed, 2009, http://www.si-folkesundhed.dk/Udgivelser/B\%C3\%B8ger\%20 og\%20rapporter/2008/2897\%20Folkesundhedsrapporten $\% 202$ 007.aspx.

[5] S. Hogg-Johnson, G. van der Velde, L. J. Carroll et al., "The burden and determinants of neck pain in the general population: results of the Bone and Joint Decade 2000-2010 Task Force on Neck Pain and Its Associated Disorders," Spine, vol. 33, supplement 4, pp. S39-S51, 2008.

[6] L. J. Carroll, L. W. Holm, S. Hogg-Johnson et al., "Course and prognostic factors for neck pain in whiplash-associated disorders (WAD). Results of the Bone and Joint Decade 20002010 task force on Neck Pain and Its Associated Disorders," 
Journal of Manipulative and Physiological Therapeutics, vol. 32, supplement 2, pp. S97-S107, 2009.

[7] A. Berglund, L. Bodin, I. Jensen, A. Wiklund, and L. Alfredsson, "The influence of prognostic factors on neck pain intensity, disability, anxiety and depression over a 2-year period in subjects with acute whiplash injury," Pain, vol. 125, no. 3, pp. 244-256, 2006.

[8] K. Boersma and S. J. Linton, "Expectancy, fear and pain in the prediction of chronic pain and disability: a prospective analysis," European Journal of Pain, vol. 10, no. 6, pp. 551-557, 2006.

[9] R. Fejer and J. Hartvigsen, "Neck pain and disability due to neck pain: what is the relation?" European Spine Journal, vol. 17, no. 1, pp. 80-88, 2008.

[10] N. Harvey and C. Cooper, "Physiotherapy for neck and back pain," British Medical Journal, vol. 330, no. 7482, pp. 53-54, 2005.

[11] A. Soderlund and P. Lindberg, "Long-term functional and psychological problems in whiplash associated disorders," International Journal of Rehabilitation Research, vol. 22, no. 2, pp. 77$84,1999$.

[12] J. Borghouts, H. Janssen, B. Koes, J. Muris, J. Metsemakers, and L. Bouter, "The management of chronic neck pain in general practice. A retrospective study," Scandinavian Journal of Primary Health Care, vol. 17, no. 4, pp. 215-220, 1999.

[13] B. G. Haraldsson, A. R. Gross, C. D. Myers et al., "Massage for mechanical neck disorders," Cochrane Database of Systematic Reviews, no. 3, Article ID CD004871, 2006.

[14] A. Gross, M. Forget, K. St George et al., "Patient education for neck pain," Cochrane Database of Systematic Reviews, no. 3, Article ID CD005106, 2012.

[15] P. Kroeling, A. Gross, C. H. Goldsmith et al., "Electrotherapy for neck pain," Cochrane Database of Systematic Reviews, no. 4 , Article ID CD004251, 2009.

[16] N. Graham, A. Gross, C. H. Goldsmith et al., "Mechanical traction for neck pain with or without radiculopathy," Cochrane Database of Systematic Reviews, no. 3, Article ID CD006408, 2008.

[17] A. P. Verhagen, G. G. Scholten-Peeters, S. van Wijngaarden, R. A. de Bie, and S. M. Bierma-Zeinstra, "Conservative treatments for whiplash," Cochrane Database of Systematic Reviews, no. 2, Article ID CD003338, 2007.

[18] A. Gross, J. Miller, J. D’Sylva et al., "Manipulation or mobilisation for neck pain: a Cochrane Review," Manual Therapy, vol. 15, no. 4, pp. 315-333, 2010.

[19] T. M. Kay, A. Gross, C. Goldsmith et al., "Exercises for mechanical neck disorders," Cochrane Database of Systematic Reviews, no. 3, Article ID CD004250, 2005.

[20] G. H. Guyatt, A. D. Oxman, G. E. Vist et al., "GRADE: an emerging consensus on rating quality of evidence and strength of recommendations," British Medical Journal, vol. 336, no. 7650, pp. 924-926, 2008.

[21] I. Kuorinka, B. Jonsson, A. Kilbom et al., "Standardised Nordic questionnaires for the analysis of musculoskeletal symptoms," Applied Ergonomics, vol. 18, no. 3, pp. 233-237, 1987.

[22] R. Christensen and H. Bliddal, "Is Phytalgic a goldmine for osteoarthritis patients or is there something fishy about this nutraceutical? A summary of findings and risk-of-bias assessment," Arthritis Research and Therapy, vol. 12, no. 1, article 105, 2010.

[23] J. P. T. Higgins and S. Green, Cochrane handbook for systematic reviews of interventions Version 5.0.1, The Cochrane Collaboration, 2008, http://www.cochrane-handbook.org.
[24] A. Liberati, D. G. Altman, J. Tetzlaff et al., "The PRISMA statement for reporting systematic reviews and meta-analyses of studies that evaluate health care interventions: explanation and elaboration," Annals of Internal Medicine, vol. 151, no. 4, pp. W65-W-94, 2009.

[25] E. M. Bartels, "How to keep up with medical literature," Best Practice and Research, vol. 23, no. 2, pp. 281-290, 2009.

[26] D. N. Resnick, "Subjective outcome assessments for cervical spine pathology: a narrative review," Journal of Chiropractic Medicine, vol. 4, no. 3, pp. 113-134, 2005.

[27] J. E. Ware Jr., "SF-36 health survey update," Spine, vol. 25, no. 24, pp. 3130-3139, 2000.

[28] C. H. P. de Koning, S. P. van den Heuvel, J. B. Staal, B. C. M. Smits-Engelsman, and E. J. M. Hendriks, "Clinimetric evaluation of methods to measure muscle functioning in patients with non-specific neck pain: a systematic review," BMC Musculoskeletal Disorders, vol. 9, article 142, 2008.

[29] C. H. P. de Koning, S. P. van den Heuvel, J. B. Staal, B. C. M. Smits-Engelsman, and E. J. M. Hendriks, "Clinimetric evaluation of active range of motion measures in patients with nonspecific neck pain: a systematic review," European Spine Journal, vol. 17, no. 7, pp. 905-921, 2008.

[30] B. Juul-Kristensen, R. Kadefors, K. Hansen, P. Byström, L. Sandsjö, and G. Sjøgaard, "Clinical signs and physical function in neck and upper extremities among elderly female computer users: the NEW study," European Journal of Applied Physiology, vol. 96, no. 2, pp. 136-145, 2006.

[31] A. C. V. Cunha, T. N. Burke, F. J. R. França, and A. P. Marques, "Effect of global posture reeducation and of static stretching on pain, range of motion, and quality of life in women with chronic neck pain: a randomized clinical trial," Clinics, vol. 63, no. 6, pp. 763-770, 2008.

[32] L. Dellve, L. Ahlstrom, A. Jonsson et al., "Myofeedback training and intensive muscular strength training to decrease pain and improve work ability among female workers on long-term sick leave with neck pain: a randomized controlled trial," International Archives of Occupational and Environmental Health, vol. 84, no. 3, pp. 335-346, 2011.

[33] D. Falla, G. Jull, P. Hodges, and B. Vicenzino, "An endurancestrength training regime is effective in reducing myoelectric manifestations of cervical flexor muscle fatigue in females with chronic neck pain," Clinical Neurophysiology, vol. 117, no. 4, pp. $828-837,2006$

[34] C. Griffiths, K. Dziedzic, J. Waterfield, and J. Sim, "Effectiveness of specific neck stabilization exercises or a general neck exercise program for chronic neck disorders: a randomized controlled trial," The Journal of Rheumatology, vol. 36, no. 2, pp. 390-397, 2009.

[35] C. Gustavsson, E. Denison, and L. V. Koch, "Self-management of persistent neck pain: a randomized controlled trial of a multi-component group intervention in primary health care," European Journal of Pain, vol. 14, no. 6, pp. 630.e1-630.e11, 2010.

[36] A. Häkkinen, H. Kautiainen, P. Hannonen, and J. Ylinen, "Strength training and stretching versus stretching only in the treatment of patients with chronic neck pain: a randomized one-year follow-up study," Clinical Rehabilitation, vol. 22, no. 7, pp. 592-600, 2008.

[37] A. Jordan, T. Bendix, H. Nielsen, F. R. Hansen, D. Høst, and A. Winkel, "Intensive training, physiotherapy, or manipulation for patients with chronic neck pain: a prospective, single-blinded, randomized clinical trial," Spine, vol. 23, no. 3, pp. 311-319, 1998. 
[38] G. Jull, D. Falla, J. Treleaven, P. Hodges, and B. Vicenzino, "Retraining cervical joint position sense: the effect of two exercise regimes," Journal of Orthopaedic Research, vol. 25, no. 3, pp. 404-412, 2007.

[39] G. A. Jull, D. Falla, B. Vicenzino, and P. W. Hodges, “The effect of therapeutic exercise on activation of the deep cervical flexor muscles in people with chronic neck pain," Manual Therapy, vol. 14, no. 6, pp. 696-701, 2009.

[40] S. O'Leary, D. Falla, P. W. Hodges, G. Jull, and B. Vicenzino, "Specific therapeutic exercise of the neck induces immediate local hypoalgesia," Journal of Pain, vol. 8, no. 11, pp. 832-839, 2007.

[41] A. Randløv, M. Østergaard, C. Manniche et al., "Intensive dynamic training for females with chronic neck/shoulder pain. A randomized controlled trial," Clinical Rehabilitation, vol. 12, no. 3, pp. 200-210, 1998.

[42] M. Revel, M. Minguet, P. Gergoy, J. Vaillant, and J. L. Manuel, "Changes in cervicocephalic kinesthesia after a proprioceptive rehabilitation program in patients with neck pain: a randomized controlled study," Archives of Physical Medicine and Rehabilitation, vol. 75, no. 8, pp. 895-899, 1994.

[43] S. Taimela, E. P. Takala, T. Asklöf, K. Seppälä, and S. Parviainen, "Active treatment of chronic neck pain: a prospective randomized intervention," Spine, vol. 25, no. 8, pp. 1021-1027, 2000.

[44] M. Viljanen, A. Malmivaara, J. Uitti, M. Rinne, P. Palmroos, and P. Laippala, "Effectiveness of dynamic muscle training, relaxation training, or ordinary activity for chronic neck pain: randomised controlled trial," British Medical Journal, vol. 327, no. 7413, pp. 475-477, 2003.

[45] F. Vonk, A. P. Verhagen, J. W. Twisk, A. J. A. Köke, M. W. C. T. Luiten, and B. W. Koes, "Effectiveness of a behaviour graded activity program versus conventional exercise for chronic neck pain patients," European Journal of Pain, vol. 13, no. 5, pp. 533541, 2009.

[46] K. Waling, G. Sundelin, C. Ahlgren, and B. Järvholm, "Perceived pain before and after three exercise programs-a controlled clinical trial of women with work-related trapezius myalgia," Pain, vol. 85, no. 1-2, pp. 201-207, 2000.

[47] J. Ylinen, E. P. Takala, M. Nykanen et al., "Active neck muscle training in the treatment of chronic neck pain in women: a randomized controlled trial," The Journal of the American Medical Association, vol. 289, no. 19, pp. 2509-2516, 2003.

[48] J. Ylinen, H. Kautiainen, K. Wirén, and A. Häkkinen, "Stretching exercises vs manual therapy in treatment of chronic neck pain: a randomized, controlled cross-over trial," Journal of Rehabilitation Medicine, vol. 39, no. 2, pp. 126-132, 2007.

[49] C. Ehrenborg and B. Archenholtz, "Is surface EMG biofeedback an effective training method for persons with neck and shoulder complaints after whiplash-associated disorders concerning activities of daily living and pain-a randomized controlled trial," Clinical Rehabilitation, vol. 24, no. 8, pp. 715-726, 2010.

[50] D. Fitz-Ritson, "Phasic exercises for cervical rehabilitation after "whiplash" trauma," Journal of Manipulative and Physiological Therapeutics, vol. 18, no. 1, pp. 21-24, 1995.

[51] G. Jull, M. Sterling, J. Kenardy, and E. Beller, "Does the presence of sensory hypersensitivity influence outcomes of physical rehabilitation for chronic whiplash?- - a preliminary RCT," Pain, vol. 129, no. 1-2, pp. 28-34, 2007.

[52] U. Pato, G. di Stefano, N. Fravi et al., "Comparison of randomized treatments for late whiplash,” Neurology, vol. 74, no. 15, pp. 1223-1230, 2010.
[53] J. M. Ryan, Reducing Pain and Disability for Whipiash Victims: A Double-Blind Randomised Controlled trial, vol. 1, Australian National University, 2002.

[54] A. Söderlund and P. Lindberg, "Cognitive behavioural components in physiotherapy management of chronic whiplash associated disorders (WAD) - a randomised group study," Physiotherapy Theory and Practice, vol. 17, no. 4, pp. 229-238, 2001.

[55] M. J. Stewart, C. G. Maher, K. M. Refshauge, R. D. Herbert, N. Bogduk, and M. Nicholas, "Randomized controlled trial of exercise for chronic whiplash-associated disorders," Pain, vol. 128, no. 1-2, pp. 59-68, 2007.

[56] G. Bronfort, R. Evans, B. Nelson, P. D. Aker, C. H. Goldsmith, and H. Vernon, "A randomized clinical trial of exercise and spinal manipulation for patients with chronic neck pain," Spine, vol. 26, no. 7, pp. 788-797, 2001.

[57] H. M. C. Lau, T. T. Wing Chiu, and T. H. Lam, "The effectiveness of thoracic manipulation on patients with chronic mechanical neck pain-a randomized controlled trial," Manual Therapy, vol. 16, no. 2, pp. 141-147, 2011.

[58] J. Martel, C. Dugas, J. D. Dubois, and M. Descarreaux, "A randomised controlled trial of preventive spinal manipulation with and without a home exercise program for patients with chronic neck pain," BMC Musculoskeletal Disorders, vol. 12, article 41, 2011.

[59] K. J. Sherman, D. C. Cherkin, R. J. Hawkes, D. L. Miglioretti, and R. A. Deyo, "Randomized trial of therapeutic massage for chronic neck pain," Clinical Journal of Pain, vol. 25, no. 3, pp. 233-238, 2009.

[60] R. Sillevis, J. Cleland, M. Hellman, and K. Beekhuizen, "Immediate effects of a thoracic spine thrust manipulation on the autonomic nervous system: a randomized clinical trial," Journal of Manual and Manipulative Therapy, vol. 18, no. 4, pp. 181-190, 2010.

[61] N. Yağci, F. Uygur, and N. Bek, "Comparison of connective tissue massage and spray-and-stretch technique in the treatment of chronic cervical myofascial pain syndrome," The Pain Clinic, vol. 16, no. 4, pp. 469-474, 2004.

[62] L. Altan, U. Bingöl, M. Aykaç, and M. Yurtkuran, "Investigation of the effect of GaAs laser therapy on cervical myofascial pain syndrome," Rheumatology International, vol. 25, no. 1, pp. 23-27, 2005.

[63] T. T. W. Chiu, C. W. Y. Hui-Chan, and G. Cheing, "A randomized clinical trial of TENS and exercise for patients with chronic neck pain," Clinical Rehabilitation, vol. 19, no. 8, pp. 850-860, 2005.

[64] R. T. Chow, G. Z. Heller, and L. Barnsley, "The effect of $300 \mathrm{~mW}$, $830 \mathrm{~nm}$ laser on chronic neck pain: a double-blind, randomized, placebo-controlled study," Pain, vol. 124, no. 1-2, pp. 201-210, 2006.

[65] U. Dundar, D. Evcik, F. Samli, H. Pusak, and V. Kavuncu, "The effect of gallium arsenide aluminum laser therapy in the management of cervical myofascial pain syndrome: a double blind, placebo-controlled study," Clinical Rheumatology, vol. 26, no. 6, pp. 930-934, 2007.

[66] M. Esenyel, N. Caglar, and T. Aldemir, "Treatment of myofascial pain," American Journal of Physical Medicine and Rehabilitation, vol. 79, no. 1, pp. 48-52, 2000.

[67] A. N. Gam, S. Warming, L. H. Larsen et al., "Treatment of myofascial trigger-points with ultrasound combined with massage and exercise-a randomised controlled trial," Pain, vol. 77, no. 1, pp. 73-79, 1998.

[68] A. Gur, A. J. Sarac, R. Cevik, O. Altindag, and S. Sarac, "Efficacy of $904 \mathrm{~nm}$ Gallium Arsenide low level laser therapy in the 
management of chronic myofascial pain in the neck: a doubleblind and randomize-controlled trial," Lasers in Surgery and Medicine, vol. 35, no. 3, pp. 229-235, 2004.

[69] F. Özdemir, M. Birtane, and S. Kokino, "The clinical efficacy of low-power laser therapy on pain and function in cervical osteoarthritis," Clinical Rheumatology, vol. 20, no. 3, pp. 181-184, 2001.

[70] U. Seidel and C. Uhlemann, "A randomised controlled doubleblind trial comparing dosed lasertherapy on acupuncture points and acupuncture for chronic cervical syndrome," Deutsche Zeitschrift fur Akupunktur, vol. 45, no. 4, pp. 258-269, 2002.

[71] N. Smania, E. Corato, A. Fiaschi, P. Pietropoli, S. M. Aglioti, and M. Tinazzi, "Repetitive magnetic stimulation a novel therapeutic approach for myofascial pain syndrome," Journal of Neurology, vol. 252, no. 3, pp. 307-314, 2005.

[72] H. Thorsen, A. N. Gam, B. H. Svensson et al., "Low level laser therapy for myofascial pain in the neck and shoulder girdle. A double-blind, cross-over study," Scandinavian Journal of Rheumatology, vol. 21, no. 3, pp. 139-141, 1992.

[73] J. M. Bland and D. G. Altman, "Comparisons within randomised groups can be very misleading," British Medical Journal, vol. 342, article d561, 2011.

[74] I. Boutron, S. Dutton, P. Ravaud, and D. G. Altman, "Reporting and interpretation of randomized controlled trials with statistically nonsignificant results for primary outcomes," The Journal of the American Medical Association, vol. 303, no. 20, pp. 20582064, 2010.

[75] J. P. Higgins, D. G. Altman, P. C. Gotzsche et al., "The Cochrane Collaboration's tool for assessing risk of bias in randomised trials," British Medical Journal, vol. 343, article d5928, 2011.

[76] C. A. Moyer, "Between-groups study designs demand betweengroups analyses: a response to hernandez-reif, shor-posner, baez, soto, mendoza, castillo, quintero, perez, and zhang," Evidence-based Complementary and Alternative Medicine, vol. 6, no. 1, pp. 49-50, 2009.

[77] P. M. Barton and K. C. Hayes, "Neck flexor muscle strength, efficiency, and relaxation times in normal subjects and subjects with unilateral neck pain and headache," Archives of Physical Medicine and Rehabilitation, vol. 77, no. 7, pp. 680-687, 1996.

[78] D. Falla, A. Rainoldi, R. Merletti, and G. Jull, "Myoelectric manifestations of sternocleidomastoid and anterior scalene muscle fatigue in chronic neck pain patients," Clinical Neurophysiology, vol. 114, no. 3, pp. 488-495, 2003.

[79] A. Jordan, J. Mehlsen, and K. Ostergaard, "A comparison of physical characteristics between patients seeking treatment for neck pain and age-matched healthy people," Journal of Manipulative and Physiological Therapeutics, vol. 20, no. 7, pp. 468-475, 1997.

[80] J. Ylinen, P. Salo, M. Nykänen, H. Kautiainen, and A. Häkkinen, "Decreased isometric neck strength in women with chronic neck pain and the repeatability of neck strength measurements," Archives of Physical Medicine and Rehabilitation, vol. 85, no. 8, pp. 1303-1308, 2004.

[81] R. T. Chow, M. I. Johnson, R. A. Lopes-Martins, and J. M. Bjordal, "Efficacy of low-level laser therapy in the management of neck pain: a systematic review and meta-analysis of randomised placebo or active-treatment controlled trials," The Lancet, vol. 374, no. 9705, pp. 1897-1908, 2009.

[82] R. W. Teasell, J. A. McClure, D. Walton et al., "A research synthesis of therapeutic interventions for whiplash-associated disorder (WAD): part 4-noninvasive interventions for chronic
WAD," Pain Research and Management, vol. 15, no. 5, pp. 313$322,2010$.

[83] R. D. Herbert and K. Bo, "Analysis of quality of interventions in systematic reviews," British Medical Journal, vol. 331, no. 7515, pp. 507-509, 2005. 


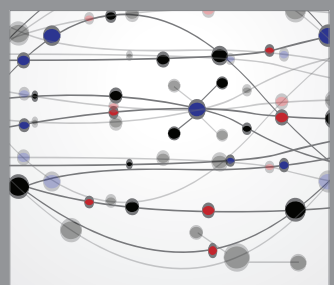

The Scientific World Journal
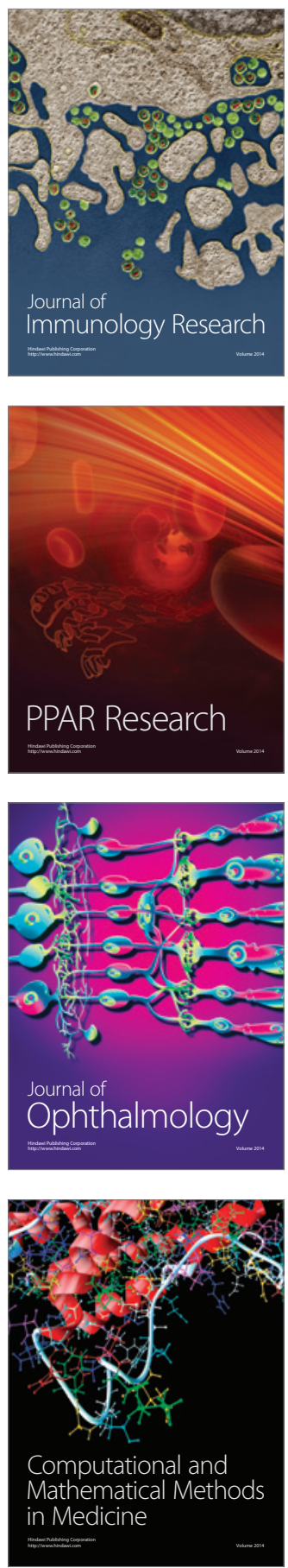

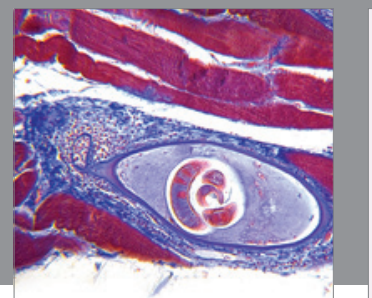

Gastroenterology

Research and Practice
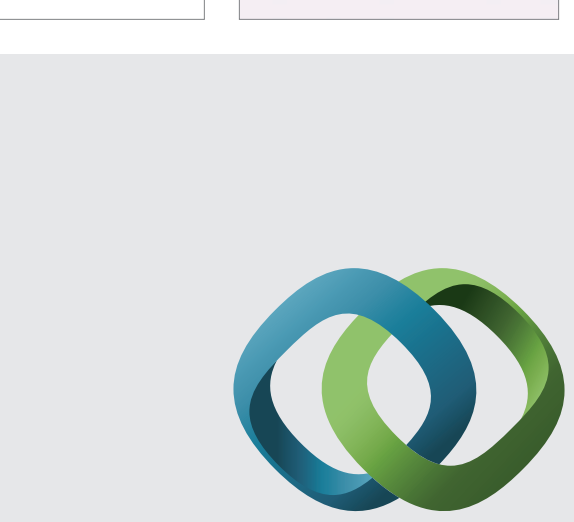

\section{Hindawi}

Submit your manuscripts at

http://www.hindawi.com
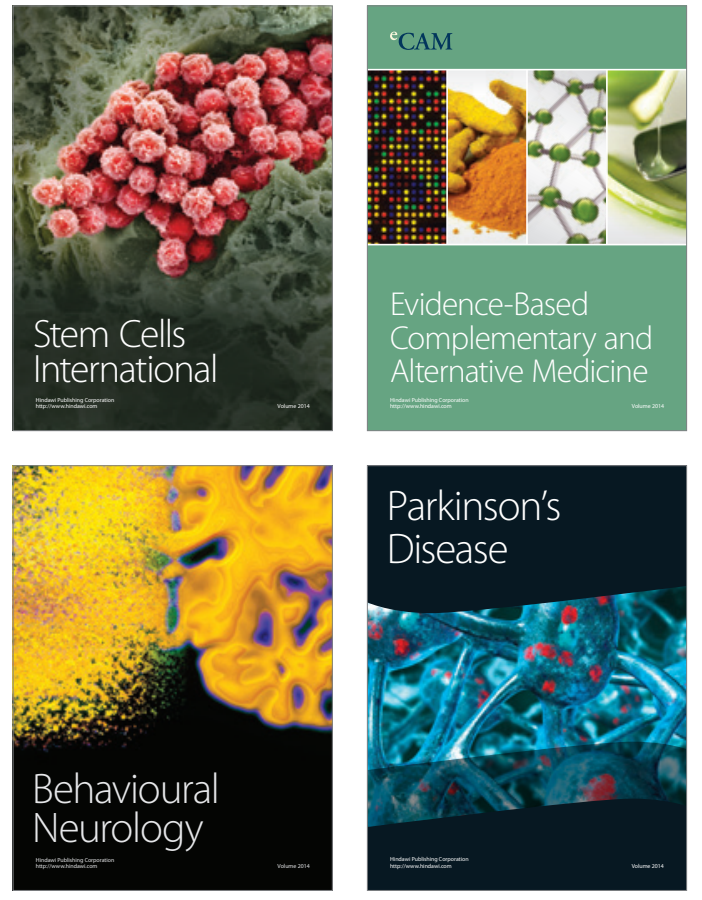
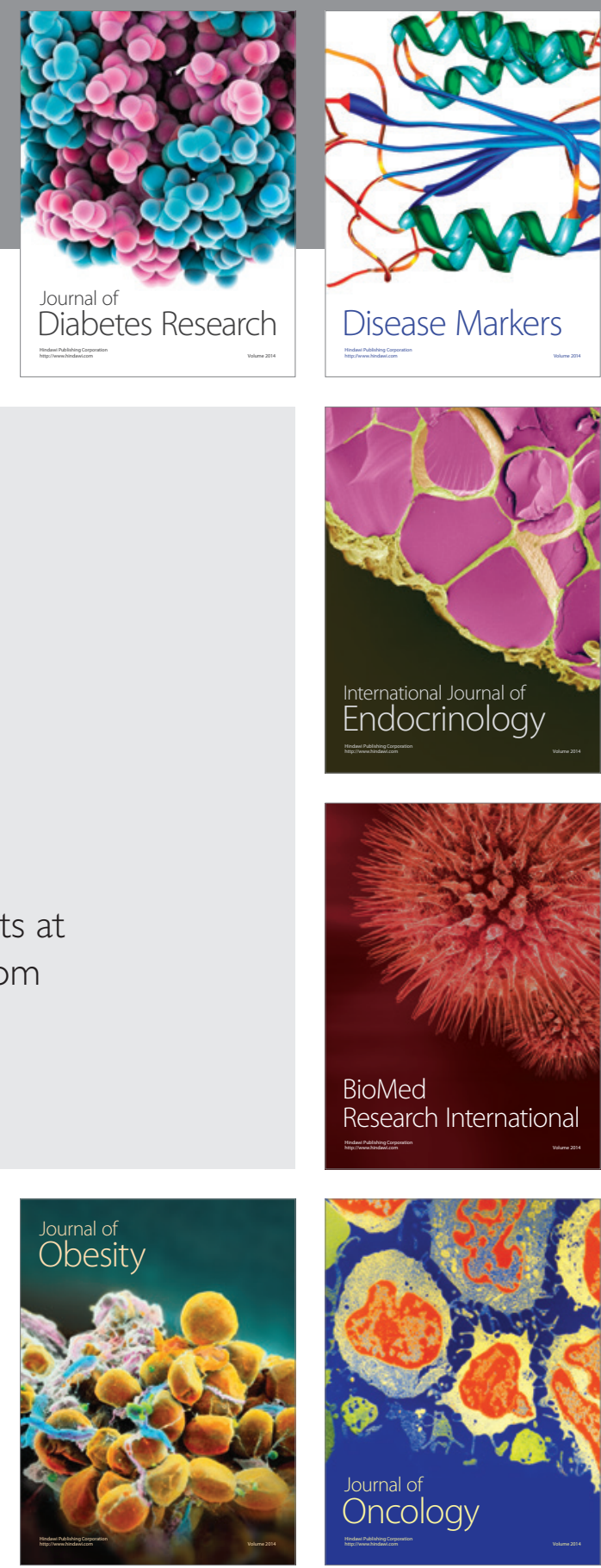

Disease Markers
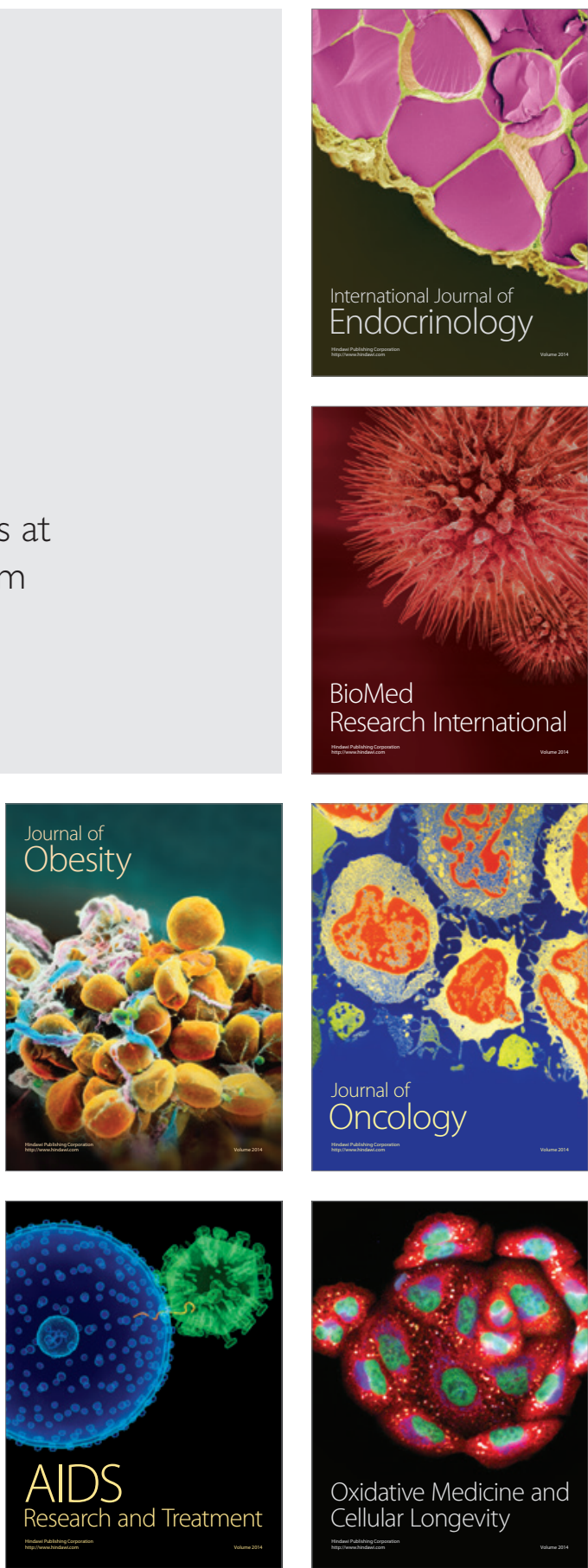